\title{
On Transitive Systems of Subspaces in a Hilbert Space
}

\author{
Yuliya P. MOSKALEVA ${ }^{\dagger}$ and Yurii S. SAMOILLENKO \\ † Taurida National University, 4 Vernads'kyi Str., Simferopol, 95007 Ukraine \\ E-mail: YulMosk@mail.ru \\ $¥$ Institute of Mathematics, National Academy of Sciences of Ukraine, \\ 3 Tereshchenkivs'ka Str., Kyiv-4, 01601 Ukraine \\ E-mail:yurii_sam@imath.kiev.ua
}

Received February 27, 2006; Published online April 12, 2006

Original article is available at http://www.emis.de/journals/SIGMA/2006/Paper042/

\section{Submitted by Anatoly Klimyk}

\begin{abstract}
Methods of $*$-representations in Hilbert space are applied to study of systems of $n$ subspaces in a linear space. It is proved that the problem of description of $n$-transitive subspaces in a finite-dimensional linear space is $*$-wild for $n \geq 5$.
\end{abstract}

Key words: algebras generated by projections; irreducible inequivalent representations; transitive nonisomorphic systems of subspaces

2000 Mathematics Subject Classification: 47A62; 16G20

\section{Introduction}

Systems of $n$ subspaces $H_{1}, H_{2}, \ldots, H_{n}$ of a Hilbert space $H$, denoted in the sequel by $S=$ $\left(H ; H_{1}, H_{2}, \ldots, H_{n}\right)$, is a mathematical object that traditionally draws an interest both by itself $[1,4,5,6]$ and in connection with the discussion on whether there exists a deeper connection between this object and the famous H. Weyl problem, the Coxeter groups, singularity theory, and physical applications.

Systems of subspaces that can be regarded as candidates for being the simplest building blocks for arbitrary systems of subspaces are those that are indecomposable or transitive $[4,5,6]$. A description of transitive and indecomposable systems is carried out up to an isomorphism of the systems of subspaces. For a description of transitive and indecomposable systems of two subspaces of a Hilbert space, as well as for transitive and indecomposable triples of a finite dimensional linear space, see, e.g., [6]. For an infinite dimensional space, not only the problem of description but even the problem of existence of transitive and indecomposable triples of subspaces is an unsolved problem [2]. For a finite dimensional linear space, transitive quadruples of subspaces are described in [3], and [4,5] give indecomposable quadruples. Examples of nonisomorphic transitive and indecomposable systems of four subspaces in an infinite dimensional space can be found, e.g., in [6].

In [6] the authors make a conjecture that there is a connection between systems of $n$ subspaces and representations of $*$-algebras that are generated by the projections, - "There seems to be interesting relations of systems of $n$-subspaces with the study of representations of $*$-algebras generated by idempotents by S. Kruglyak, V. Ostrovskyi, V. Rabanovich, Yu. Samoílenko and other. But we do not know the exact implication ...". The present article deals with this implication.

Let us consider systems of subspaces of the form $S_{\pi}=\left(H ; P_{1} H, P_{2} H, \ldots, P_{n} H\right)$, where the orthogonal projections $P_{1}, P_{2}, \ldots, P_{n}$ make a $*$-representation $\pi$ of the $*$-algebra generated by the 
projections, and $H$ is the representation space. For the $*$-algebras $\mathcal{P}_{4, \mathrm{com}}=\mathbb{C}\left\langle p_{1}, p_{2}, p_{3}, p_{4}\right| p_{k}^{2}=$

$\left.p_{k}^{*}=p_{k},\left[\sum_{k=1}^{4} p_{k}, p_{i}\right]=0, \forall i=1,2,3,4\right\rangle$, it was proved in [11] that irreducible inequivalent *-representations $\pi$ of the $*$-algebra $\mathcal{P}_{4 \text {,com }}$ make a complete list of nonisomorphic transitive quadruples of subspaces $S_{\pi}$ of a finite dimensional linear space.

In this paper, we make an analysis of complexity of the description problem for transitive systems of subspaces $S=\left(H ; H_{1}, H_{2}, \ldots, H_{n}\right)$ for $n \geq 5$. In Section 3 , we prove that it is an extremely difficult problem to describe nonisomorphic transitive quintuples of subspaces $S=\left(H ; P_{1} H, P_{2} H, \ldots, P_{5} H\right)$ even under the assumption that the sum of the corresponding five projections equals $2 I$; in other words, the problem of describing inequivalent $*$-representations of the $*$-algebras that give rise to nonisomorphic transitive systems, is $*$-wild.

Since the problem of describing the system of $n$ subspaces up to an isomorphism is complicated, it seems natural to describe transitive systems that correspond to $*$-representations of various algebras generated by projections (Sections 4 and 5).

In Section 4, we consider transitive systems $S_{\pi}$ of $n$ subspaces, where $\pi \in \operatorname{Rep} \mathcal{P}_{n, \alpha}, \mathcal{P}_{n, \alpha}=$ $\mathbb{C}\left\langle p_{1}, p_{2}, \ldots, p_{n} \mid p_{1}+p_{2}+\cdots+p_{n}=\alpha e, p_{j}^{2}=p_{j}, p_{j}^{*}=p_{j}, \forall j=1, \ldots, n\right\rangle$, and $\alpha$ takes values in a fixed set. In Section 5, using nonisomorphic transitive systems $S_{\pi}$ of $n$ subspaces, where $\pi$ belongs to $\operatorname{Rep} \mathcal{P}_{n, \alpha}$, we construct nonisomorphic transitive systems $S_{\hat{\pi}}$ of $n+1$ subspaces, where $\hat{\pi}$ is in $\operatorname{Rep} \mathcal{P}_{n, \text { abo }, \tau}, \mathcal{P}_{n, \text { abo }, \tau}=\mathbb{C}\left\langle q_{1}, q_{2}, \ldots, q_{n}, p\right| q_{1}+q_{2}+\cdots+q_{n}=e, q_{j} p q_{j}=\tau q_{j}$, $\left.q_{j}^{2}=q_{j}, q_{j}^{*}=q_{j}, \forall j=1, \ldots, n, p^{2}=p, p^{*}=p\right\rangle$.

\section{Definitions and main properties}

In this section we make necessary definitions and recall known facts; the proofs can be found in $[6,9]$. Let $H$ be a Hilbert space and $H_{1}, H_{2}, \ldots, H_{n}$ be $n$ subspaces of $H$. Denote by $S=\left(H ; H_{1}, H_{2}, \ldots, H_{n}\right)$ the system of $n$ subspaces of the space $H$. Let $S=\left(H ; H_{1}, H_{2}, \ldots, H_{n}\right)$ be a system of $n$ subspaces of a Hilbert space $H$ and $\tilde{S}=\left(\tilde{H} ; \tilde{H}_{1}, \tilde{H}_{2}, \ldots, \tilde{H}_{n}\right)$ a system of $n$ subspaces of a Hilbert space $\tilde{H}$. A linear map $R: H \rightarrow \tilde{H}$ from the space $H$ to the space $\tilde{H}$ is called a homomorphism of the system $S$ into the system $\tilde{S}$ and denoted by $R: S \rightarrow \tilde{S}$, if $R\left(H_{i}\right) \subset \tilde{H}_{i}, i=1, \ldots, n$. A homomorphism $R: S \rightarrow \tilde{S}$ of a system $S$ into a system $\tilde{S}$ is called an isomorphism, $R: S \rightarrow \tilde{S}$, if the mapping $R: H \rightarrow \tilde{H}$ is a bijection and $R\left(H_{i}\right)=\tilde{H}_{i}$, $\forall i=1, \ldots, n$. Systems $S$ and $\tilde{S}$ will be called isomorphic, denoted by $S \cong \tilde{S}$, if there exists an isomorphism $R: S \rightarrow \tilde{S}$.

Denote by $\operatorname{Hom}(S, \tilde{S})$ the set of homomorphisms of a system $S$ into a system $\tilde{S}$ and by $\operatorname{End}(S):=\operatorname{Hom}(S, S)$ the algebra of endomorphisms of $S$ into $S$, that is,

$$
\operatorname{End}(S)=\left\{R \in B(H) \mid R\left(H_{i}\right) \subset H_{i}, i=1, \ldots, n\right\} .
$$

A system $S=\left(H ; H_{1}, H_{2}, \ldots, H_{n}\right)$ of $n$ subspaces of a Hilbert space $H$ is called transitive, if $\operatorname{End}(S)=\mathbb{C} I_{H}$

Denote

$$
\operatorname{Idem}(S)=\left\{R \in B(H) \mid R\left(H_{i}\right) \subset H_{i}, i=1, \ldots, n, R^{2}=R\right\}
$$

A system $S=\left(H ; H_{1}, H_{2}, \ldots, H_{n}\right)$ of $n$ subspaces of a space $H$ is called indecomposable, if $\operatorname{Idem}(S)=\left\{0, I_{H}\right\}$.

Isomorphic systems are either simultaneously transitive or intransitive, decomposable or indecomposable. We say that $S \cong \tilde{S}$ up to permutation of subspaces, if there exists a permutation $\sigma \in$ $S_{n}$ such that the systems $\sigma(S)$ and $\tilde{S}$ are isomorphic, where $\sigma(S)=\left(H ; H_{\sigma(1)}, H_{\sigma(2)}, \ldots, H_{\sigma(n)}\right)$, so that there exists an invertible operator $R: H \rightarrow \tilde{H}$ such that $R\left(H_{\sigma(i)}\right)=\tilde{H}_{i}, \forall i=1, \ldots, n$. 
Let us now recall the notion of unitary equivalence for systems and collections of orthogonal projections. Systems $S$ and $\tilde{S}$ are called unitary equivalent, or simply equivalent, if $S \cong \widetilde{S}$ and it is possible to choose the isomorphism $R: S \rightarrow \tilde{S}$ to be a unitary operator.

To every system $S=\left(H ; H_{1}, H_{2}, \ldots, H_{n}\right)$ of $n$ subspaces of a Hilbert space $H$, one can naturally associate a system of orthogonal projections $P_{1}, P_{2}, \ldots, P_{n}$, where $P_{i}$ is the orthogonal projection operator onto the space $H_{i}, i=1, \ldots, n$. A system of projections $P_{1}, P_{2}, \ldots, P_{n}$ on a Hilbert space $H$ such that $\operatorname{Im} P_{i}=H_{i}$ for $i=1, \ldots, n$ is called a system of orthogonal projections associated to the system of subspaces, $S=\left(H ; H_{1}, H_{2}, \ldots, H_{n}\right)$. Conversely, to each system of projections there naturally corresponds a system of subspaces. A system $S=\left(H ; P_{1} H, P_{2} H, \ldots, P_{n} H\right)$ is called a system corresponding to the system of projections $P_{1}, P_{2}, \ldots, P_{n}$.

A system of orthogonal projections $P_{1}, P_{2}, \ldots, P_{n}$ on a Hilbert space $H$ is called unitary equivalent to a system $\tilde{P}_{1}, \tilde{P}_{2}, \ldots, \tilde{P}_{n}$ on a Hilbert space $\tilde{H}$, if there exists a unitary operator $R: H \rightarrow \tilde{H}$ such that $R P_{i}=\tilde{P}_{i} R, i=1, \ldots, n$. Systems $S$ and $\tilde{S}$ are unitary equivalent if and only if the corresponding systems of orthogonal projections are unitary equivalent.

A system of orthogonal projections $P_{1}, P_{2}, \ldots, P_{n}$ on a Hilbert space $H$ is called irreducible if zero and $H$ are the only invariant subspaces. Unitary equivalent systems of orthogonal projections are both either reducible or irreducible.

If systems $S$ and $\tilde{S}$ are unitary equivalent, then $S \cong \tilde{S}$. The converse is not true.

Example 1. Let $S=\left(\mathbb{C}^{2} ; \mathbb{C}(1,0), \mathbb{C}(\cos \theta, \sin \theta)\right), \theta \in(0, \pi / 2)$, and $\tilde{S}=\left(\mathbb{C}^{2} ; \mathbb{C}(1,0), \mathbb{C}(0,1)\right)$. The decomposable system $S$ that corresponds to an irreducible pair of orthogonal projections, is isomorphic but not unitary equivalent to the decomposable system $\tilde{S}$ that corresponds to a reducible pair of orthogonal projections.

Finally, let us mention the relationship between the notions of transitivity, indecomposability, and irreducibility. If a system of subspaces is transitive, then it is indecomposable, but not vice versa. Indecomposability of a system of subspaces implies irreducibility of the corresponding system of orthogonal projections, but not conversely.

\section{On $*$-wildness of the description problem for transitive systems of $n$ subspaces for $n \geq 5$}

\subsection{On *-wildness of the description problem for transitive systems that correspond to orthogonal projections}

A description of transitive quadruples of subspaces of a finite dimensional linear space is given in [3]. We will show that such a problem for $n$ subspaces, $n \geq 5$, is extremely complicated (*-wild).

Consider a system of five subspaces, which corresponds to the five orthogonal projections

$$
\begin{aligned}
& P_{1}=\left(\begin{array}{ll}
I & 0 \\
0 & 0
\end{array}\right), \quad P_{2}=\left(\begin{array}{ll}
0 & 0 \\
0 & I
\end{array}\right), \quad P_{3}=\frac{1}{2}\left(\begin{array}{ll}
I & I \\
I & I
\end{array}\right), \\
& P_{4}=\frac{1}{2}\left(\begin{array}{cc}
I & U \\
U^{*} & I
\end{array}\right), \quad P_{5}=\frac{1}{2}\left(\begin{array}{cc}
I & V \\
V^{*} & I
\end{array}\right)
\end{aligned}
$$

that act on the space $\mathcal{H}=H \oplus H$, where $H$ is a Hilbert space and $U$ and $V$ are unitary operators. Denote this system of subspaces by $S_{U, V}$. So, $S_{U, V}=\left(\mathcal{H} ; P_{1} \mathcal{H}, P_{2} \mathcal{H}, P_{3} \mathcal{H}, P_{4} \mathcal{H}, P_{5} \mathcal{H}\right)$. Consider the system $S_{\tilde{U}, \tilde{V}}=\left(\tilde{\mathcal{H}} ; \tilde{P}_{1} \tilde{\mathcal{H}}, \tilde{P}_{2} \tilde{\mathcal{H}}, \tilde{P}_{3} \tilde{\mathcal{H}}, \tilde{P}_{4} \tilde{\mathcal{H}}, \tilde{P}_{5} \tilde{\mathcal{H}}\right)$ that corresponds to the collection of orthogonal projections $\tilde{P}_{1}, \tilde{P}_{2}, \tilde{P}_{3}, \tilde{P}_{4}, \tilde{P}_{5}$ that have the above type and act on the space $\tilde{\mathcal{H}}=\tilde{H} \oplus \tilde{H}$; here $\tilde{H}$ is a Hilbert space and $\tilde{U}, \tilde{V}$ is a pair of unitary operators. 
Theorem 1. The system $S_{U, V}$ is transitive if and only if the unitary operators $U, V$ are irreducible. Also, $S_{U, V} \cong S_{\tilde{U}, \tilde{V}}$ if and only if the pair of unitary operators $U, V$ is unitary equivalent to the pair of unitary operators $\tilde{U}, \tilde{V}$.

Proof. Denote $H_{i}=P_{i} \mathcal{H}, i=1, \ldots, 5$. For $H_{1}$ and $H_{2}$, we have

$$
H_{1}=H \oplus 0, \quad H_{2}=0 \oplus H .
$$

For $H_{3}, H_{4}$, and $H_{5}$, respectively,

$$
H_{3}=\{(x, x) \mid x \in H\}, \quad H_{4}=\{(U x, x) \mid x \in H\}, \quad H_{5}=\{(V x, x) \mid x \in H\} .
$$

Let us prove an auxiliary identity

$$
\begin{aligned}
& \left\{\mathcal{R} \in B(\mathcal{H}, \tilde{\mathcal{H}}) \mid \mathcal{R}\left(H_{i}\right) \subset \tilde{H}_{i}, i=1, \ldots, 5\right\} \\
& \quad=\{R \oplus R \in B(\mathcal{H}, \tilde{\mathcal{H}}) \mid R \in B(H, \tilde{H}), R U=\tilde{U} R, R V=\tilde{V} R\} .
\end{aligned}
$$

The first three inclusions, $\mathcal{R}\left(H_{i}\right) \subset \tilde{H}_{i}, i=1,2,3$, imply that any operator $\mathcal{R}$ in $B(\mathcal{H}, \tilde{\mathcal{H}})$ can be represented as $\mathcal{R}=R \oplus R$, where $R \in B(H, \tilde{H})$. The fourth inclusion, $\mathcal{R}\left(H_{4}\right) \subset \tilde{H}_{4}$, implies $R U=\tilde{U} R$, and the fifth one, $\mathcal{R}\left(H_{5}\right) \subset \tilde{H}_{5}$, gives $R V=\tilde{V} R$. The converse implications finish the proof of (1).

It directly follows from (1) that $S_{U, V} \cong S_{\tilde{U} \tilde{V}}$ if and only if the pair of unitary operators $U$, $V$ is similar to the pair of unitary operators $\tilde{U}, \tilde{V}$. By [9], a pair of unitary operators $U, V$ is similar to a pair of unitary operators $\tilde{U}, \tilde{V}$ if and only if the pair of unitary operators $U, V$ is unitary equivalent to the pair of unitary operators $\tilde{U}, \tilde{V}$.

Now, setting $S_{\tilde{U}, \tilde{V}}=S_{U, V}$, rewrite the identity (1) as follows:

$$
\begin{aligned}
\operatorname{End}\left(S_{U, V}\right) & =\left\{\mathcal{R} \in B(\mathcal{H}) \mid \mathcal{R}\left(H_{i}\right) \subset H_{i}, i=1, \ldots, 5\right\} \\
& =\{R \oplus R \in B(\mathcal{H}) \mid R \in B(H), R U=U R, R V=V R\} .
\end{aligned}
$$

The latter identity immediately implies that the system $S_{U, V}$ is transitive if and only if the unitary operators $U, V$ are irreducible.

Theorem 1 allows to identify the description problem for nonisomorphic transitive quintuples that correspond to five orthogonal projections of a special type with that for inequivalent irreducible pairs of unitary operators. The latter problem is $*$-wild in the theory of $*$-representations of $*$-algebras $[8,9]$.

\subsection{On *-wildness of the description problem for transitive systems corresponding to orthogonal projections with an additional relation}

Let $P_{1}, P_{2}, P_{3}$ be orthogonal projections on a Hilbert space $H$, and $P_{2}, P_{3}$ be mutually orthogonal. Introduce a system of five subspaces of the space $H$ corresponding to the collection of orthogonal projections $P_{1}, P_{1}^{\perp}, P_{2}, P_{3},\left(P_{2}+P_{3}\right)^{\perp}$. Denote

$$
S_{P_{1}, P_{2} \perp P_{3}}=\left(H ; \operatorname{Im} P_{1}, \operatorname{Im} P_{1}^{\perp}, \operatorname{Im} P_{2}, \operatorname{Im} P_{3}, \operatorname{Im}\left(P_{2}+P_{3}\right)^{\perp}\right) .
$$

Theorem 2. Let $P_{1}, P_{2}, P_{3}$ be orthogonal projections on a Hilbert space $H$ such that $P_{2}$ and $P_{3}$ are mutually orthogonal, and $\tilde{P}_{1}, \tilde{P}_{2}, \tilde{P}_{3}$ be orthogonal projections on a Hilbert space $\tilde{H}$ such that $\tilde{P}_{2}$ and $\tilde{P}_{3}$ are mutually orthogonal. Then the system $S_{P_{1}, P_{2} \perp P_{3}}$ is transitive if and only if the projections $P_{1}, P_{2}, P_{3}$ are irreducible. Also, $S_{P_{1}, P_{2} \perp P_{3}} \cong S_{\tilde{P}_{1}, \tilde{P}_{2} \perp \tilde{P}_{3}}$ if and only if the triple of the orthogonal projections $P_{1}, P_{2}, P_{3}$ is unitary equivalent to the triple of the orthogonal projections $\tilde{P}_{1}, \tilde{P}_{2}, \tilde{P}_{3}$. 
Proof. Denote $H_{1}=\operatorname{Im} P_{1}, H_{2}=\operatorname{Im} P_{1}^{\perp}, H_{3}=\operatorname{Im} P_{2}, H_{4}=\operatorname{Im} P_{3}, H_{5}=\operatorname{Im}\left(P_{2}+P_{3}\right)^{\perp}$, and let $\tilde{H}_{1}=\operatorname{Im} \tilde{P}_{1}, \tilde{H}_{2}=\operatorname{Im} \tilde{P}_{1}^{\perp}, \tilde{H}_{3}=\operatorname{Im} \tilde{P}_{2}, \tilde{H}_{4}=\operatorname{Im} \tilde{P}_{3}, \tilde{H}_{5}=\operatorname{Im}\left(\tilde{P}_{2}+\tilde{P}_{3}\right)^{\perp}$.

The proof of the theorem directly follows from the identity

$$
\left\{R \in B(H, \tilde{H}) \mid R\left(H_{i}\right) \subset \tilde{H}_{i}, i=1, \ldots, 5\right\}=\left\{R \in B(H, \tilde{H}) \mid R P_{i}=\tilde{P}_{i} R, i=1,2,3\right\} .
$$

Theorem 2 identifies the description problem for nonisomorphic transitive quintuples of subspaces corresponding to quintuples of orthogonal projections of a special type, the ones such that their sum equals $2 I_{H}$, with that for inequivalent irreducible triples $P_{1}, P_{2}, P_{3}$ of orthogonal projections satisfying the condition $P_{2} \perp P_{3}$. The latter problem is $*$-wild in the theory of *-representations of $*$-algebras $[8,9]$.

\section{Transitive systems of subspaces corresponding to Rep $\mathcal{P}_{n, \text { com }}$}

\subsection{On $*$-representations of the $*$-algebra $\mathcal{P}_{n, \text { com }}$}

Denote by $\Sigma_{n}(n \in \mathbb{N})$ the set $\alpha \in \mathbb{R}_{+}$such that there exists at least one $*$-representation of the $*$-algebra $\mathcal{P}_{n, \alpha}=\mathbb{C}\left\langle p_{1}, p_{2}, \ldots, p_{n} \mid p_{k}^{2}=p_{k}^{*}=p_{k}, \sum_{k=1}^{n} p_{k}=\alpha e\right\rangle$, i.e., the set of real numbers $\alpha$ such that there exist $n$ orthogonal projections $P_{1}, P_{2}, \ldots, P_{n}$ on a Hilbert space $H$ satisfying $\sum_{k=1}^{n} P_{k}=\alpha I_{H}$. It follows from the definition of the algebra $\mathcal{P}_{n, \mathrm{com}}=\mathbb{C}\left\langle p_{1}, p_{2}, \ldots, p_{n}\right| p_{k}^{2}=p_{k}^{*}=$ $\left.p_{k},\left[\sum_{k=1}^{n} p_{k}, p_{i}\right]=0, \forall i=1, \ldots, n\right\rangle$ that all irreducible $*$-representations of $\mathcal{P}_{n, \text { com }}$ coincide with the union of irreducible $*$-representations of $\mathcal{P}_{n, \alpha}$ taken over all $\alpha \in \Sigma_{n}$.

A description of the set $\Sigma_{n}$ for all $n \in \mathbb{N}$ is obtained by S.A. Kruglyak, V.I. Rabanovich, and Yu.S. Samoǐlenko in [7], and is given by

$$
\begin{aligned}
& \Sigma_{2}=\{0,1,2\}, \quad \Sigma_{3}=\left\{0,1, \frac{3}{2}, 2,3\right\}, \\
& \Sigma_{n}=\left\{\Lambda_{n}^{0}, \Lambda_{n}^{1},\left[\frac{n-\sqrt{n^{2}-4 n}}{2}, \frac{n+\sqrt{n^{2}-4 n}}{2}\right], n-\Lambda_{n}^{1}, n-\Lambda_{n}^{0}\right\} \text { for } n \geq 4, \\
& \Lambda_{n}^{0}=\left\{0,1+\frac{1}{n-1}, 1+\frac{1}{(n-2)-\frac{1}{n-1}}, \ldots, 1+\frac{1}{\left.(n-2)-\frac{1}{(n-2)-\frac{1}{\ddots \cdot-\frac{1}{n-1}}}, \ldots\right\},}\right. \\
& \Lambda_{n}^{1}=\left\{1,1+\frac{1}{n-2}, 1+\frac{1}{(n-2)-\frac{1}{n-2}}, \ldots, 1+\frac{1}{(n-2)-\frac{1}{(n-2)-\frac{1}{\cdot \cdot-\frac{1}{n-2}}}}, \ldots\right\} .
\end{aligned}
$$

Here, the elements of the sets $\Lambda_{n}^{0}, \Lambda_{n}^{1}, n-\Lambda_{n}^{1}, n-\Lambda_{n}^{0}$, in what follows, will be called points of the discrete spectrum of the description problem for unitary representations of the algebra $\mathcal{P}_{n, \mathrm{com}}$, whereas the elements of the line segment $\left[\frac{n-\sqrt{n^{2}-4 n}}{2}, \frac{n+\sqrt{n^{2}-4 n}}{2}\right]$ are called point of the continuous spectrum. For each point $\alpha$ in the sets $\Lambda_{n}^{0}, n-\Lambda_{n}^{0}$ there exists, up to unitary equivalence, a unique irreducible $*$-representation of the $*$-algebra $\mathcal{P}_{n, \alpha}$ and, hence, that of $\mathcal{P}_{n, \text { com }}$. For each point $\alpha$ in the sets $\Lambda_{n}^{1}, n-\Lambda_{n}^{1}$ there exist $n$ inequivalent irreducible $*$-representations of the *-algebra $\mathcal{P}_{n, \alpha}$ and, hence, those of $\mathcal{P}_{n, \text { com }}$.

An important instrument for describing the set $\Sigma_{n}$ and representations of $\mathcal{P}_{n \text {,com }}$ is use of Coxeter functors, constructed in [7], between the categories of $*$-representations of $\mathcal{P}_{n, \alpha}$ for different values of the parameters.

Define a functor $\mathcal{T}: \operatorname{Rep} \mathcal{P}_{n, \alpha} \rightarrow \operatorname{Rep} \mathcal{P}_{n, n-\alpha}$, see [7]. Let $\pi$ be a representation of the algebra $\mathcal{P}_{n, \alpha}$, and $\pi\left(p_{i}\right)=P_{i}, i=1, \ldots, n$, be orthogonal projections on a representation space $H$. 
Then the representation $\hat{\pi}=\mathcal{T}(\pi)$ in Rep $\mathcal{P}_{n, n-\alpha}$ is defined by the identities $\hat{\pi}\left(p_{i}\right)=\left(I-P_{i}\right)$ that give orthogonal projections on $H$. We leave out a description of the action of the functor $\mathcal{T}$ on morphisms of the category $\operatorname{Rep} \mathcal{P}_{n, \alpha}$, since it is not used in the sequel. Let us now define a functor $\mathcal{S}: \operatorname{Rep} \mathcal{P}_{n, \alpha} \rightarrow \operatorname{Rep} \mathcal{P}_{n, \frac{\alpha}{\alpha-1}}$, see [7]. Again, let $\pi$ denote a representation in Rep $\mathcal{P}_{n, \alpha}$, and by $P_{1}, P_{2}, \ldots, P_{n}$ denote the corresponding orthogonal projections on the representation space $H$. Consider the subspaces $H_{i}=\operatorname{Im} P_{i}(i=1, \ldots, n)$. Let $\Gamma_{i}: H_{i} \rightarrow H, i=1, \ldots, n$, be the natural isometries. Then

$$
\Gamma_{i}^{*} \Gamma_{i}=I_{H_{i}}, \quad \Gamma_{i} \Gamma_{i}^{*}=P_{i}, \quad i=1, \ldots, n .
$$

Let an operator $\Gamma$ be defined by the matrix $\Gamma=\left[\Gamma_{1}, \Gamma_{2}, \ldots, \Gamma_{n}\right]: \mathcal{H}=H_{1} \oplus H_{2} \oplus \cdots \oplus H_{n} \rightarrow$ $H$. Then the natural isometry $\sqrt{\frac{\alpha-1}{\alpha}} \Delta^{*}$ that acts from the orthogonal complement $\hat{H}$ to the subspace $\operatorname{Im} \Gamma^{*}$ into the space $\mathcal{H}$ defines the isometries $\Delta_{k}=\left.\Delta\right|_{\operatorname{Im} P_{k}}: H_{k} \rightarrow \hat{H}, k=1, \ldots, n$. The orthogonal projections $Q_{i}=\Delta_{i} \Delta_{i}^{*}, i=1, \ldots, n$, on the space $\hat{H}$ make the corresponding representation in $\mathcal{S}\left(\operatorname{Rep} \mathcal{P}_{n, \alpha}\right)$, i.e. the representation $\hat{\pi}=\mathcal{S}(\pi)$ in $\operatorname{Rep} \mathcal{P}_{n, \frac{\alpha}{\alpha-1}}$ is given by the identities $\hat{\pi}\left(p_{i}\right)=Q_{i}$. Write down the relations satisfied by the operators $\left\{\Delta_{i}\right\}_{i}^{n}$,

$$
\Delta_{i}^{*} \Delta_{i}=I_{H_{i}}, \quad \Delta_{i} \Delta_{i}^{*}=Q_{i}, \quad i=1, \ldots, n .
$$

We will not describe the action of the functor $\mathcal{S}$ on morphisms of the category Rep $\mathcal{P}_{n, \alpha}$, since we will not use it in the sequel.

Following [7], introduce a functor $\Phi^{+}: \operatorname{Rep} \mathcal{P}_{n, \alpha} \rightarrow \operatorname{Rep} \mathcal{P}_{n, 1+\frac{1}{n-1-\alpha}}$ defined by $\Phi^{+}(\pi)=$ $\mathcal{S}(\mathcal{T}(\pi))$ for $\alpha<n-1$. Denote by $\pi_{k}(k=0,1, \ldots, n)$ the following representations in Rep $\mathcal{P}_{n, \alpha}$ : $\pi_{0}\left(p_{i}\right)=0, i=1, \ldots, n$, where the space of representation is $\mathbb{C} ; \pi_{k}\left(p_{i}\right)=0$ if $i \neq k$ and $\pi_{k}\left(p_{k}\right)=1, k=1, \ldots, n$, with $\mathbb{C}$ as the representation space. For an arbitrary irreducible representation $\pi$ of the algebra $\mathcal{P}_{n, \alpha}$ in the case of points of the discrete spectrum, one can assert that either $\pi$ or $\mathcal{T}(\pi)$ is unitary equivalent to a representation of the form $\Phi^{+s}(\check{\pi})$, where the representation $\check{\pi}$ is one of the simplest representations $\pi_{k}, k=\overline{0, n}$, and $s$ is a natural number.

\subsection{Transitive systems of $n$ subspaces corresponding to Rep $\mathcal{P}_{n, \alpha}$}

The systems of subspaces, $S_{\pi_{k}}, k=0,1, \ldots, n$, are clearly nonisomorphic transitive systems of $n$ subspaces of the space $\mathbb{C}$. I.M. Gel'fand and V.A. Ponamarev in [4], by using the functor technique, construct from the systems $S_{\pi_{k}}, k=0,1, \ldots, n$, infinite series of indecomposable systems, which turn out to be are transitive, of $n$ subspaces. In this section we show that the Coxeter functors in [7], as the functors in [4], transform nonisomorphic transitive systems into nonisomorphic transitive systems and, consequently, all systems of the form $S_{\Phi^{+s}(\tilde{\pi})}$ and $S_{\Phi^{+s}(\check{\pi})}^{\perp}$, where the representation $\check{\pi}$ is one of the simplest representations $\pi_{k}, k=0,1, \ldots, n$, and $s$ is a natural number, will be nonisomorphic transitive systems. Hence, we have the following theorem.

Theorem 3. Systems of $n$ subspaces $S_{\pi}$ constructed from irreducible inequivalent representations $\pi \in \operatorname{Rep} \mathcal{P}_{n, \alpha}$, for $\alpha$ in the discrete spectrum, are nonisomorphic and transitive.

To prove the theorem, by using the Coxeter functors $\mathcal{T}$ and $\mathcal{S}$ in [7], we construct auxiliary functors $\mathcal{T}^{\prime}$ and $\mathcal{S}^{\prime}$. The action of the functors $\mathcal{T}^{\prime}: \operatorname{Rep} \mathcal{P}_{n, \alpha} \rightarrow \operatorname{Rep} \mathcal{P}_{n, n-\alpha}$ and $\mathcal{S}^{\prime}: \operatorname{Rep} \mathcal{P}_{n, \alpha} \rightarrow$ Rep $\mathcal{P}_{n, \frac{\alpha}{\alpha-1}}$ on the objects of the category is defined to coincide with the actions of $\mathcal{T}$ and $\mathcal{S}$, that is, $\mathcal{T}(\pi)=\mathcal{T}^{\prime}(\pi)$ and $\mathcal{S}(\pi)=\mathcal{S}^{\prime}(\pi) \forall \pi \in \operatorname{Rep} \mathcal{P}_{n, \alpha}$. The morphisms of the category of representations are defined differently. Let $\pi \in \operatorname{Rep}\left(\mathcal{P}_{n, \alpha}, H\right)$ and $\tilde{\pi} \in \operatorname{Rep}\left(\mathcal{P}_{n, \alpha}, \tilde{H}\right)$. A linear 
operator $C \in B(H, \tilde{H})$ is called a morphism of the category of representations, $C \in \operatorname{Mor}(\pi, \tilde{\pi})$, if $C \pi\left(p_{i}\right)=\tilde{\pi}\left(p_{i}\right) C \pi\left(p_{i}\right), i=1, \ldots, n$, that is,

$$
C P_{i}=\tilde{P}_{i} C P_{i}, \quad i=1, \ldots, n .
$$

The restrictions $\left.C\right|_{H_{i}}, i=1, \ldots, n$, are denoted by $C_{i}$. Let us show that the operators $C_{i}$ map $H_{i}$ into $\tilde{H}_{i}$, that is,

$$
C_{i}\left(H_{i}\right) \subset \tilde{H}_{i}, \quad i=1, \ldots, n .
$$

Indeed, for $x \in H_{i}$, we have $C_{i} x=C x=C P_{i} x=\tilde{P}_{i} C P_{i} x$ and, consequently, $C_{i} x \in \tilde{H}_{i}$.

If $x \in H_{i}$, then (4) and (5) give

$$
C \Gamma_{i} x=C x=C P_{i} x=\tilde{P}_{i} C P_{i} x=\tilde{P}_{i} C_{i} x=C_{i} x=\tilde{\Gamma}_{i} C_{i} x
$$

so that

$$
C \Gamma_{i}=\tilde{\Gamma}_{i} C_{i}, \quad i=1, \ldots, n .
$$

The identities (4) are equivalent to the inclusions $C\left(H_{i}\right) \subset \tilde{H}_{i}, i=1, \ldots, n$, which immediately gives the following relations:

$$
C_{i}=\tilde{\Gamma}_{i}^{*} C \Gamma_{i}, \quad i=1, \ldots, n .
$$

Formula (6) allows to represent $C$ as

$$
C=\frac{1}{\alpha} \sum_{i=1}^{n} \tilde{\Gamma}_{i} C_{i} \Gamma_{i}^{*} .
$$

Indeed, $\frac{1}{\alpha} \sum_{i=1}^{n} \tilde{\Gamma}_{i} C_{i} \Gamma_{i}^{*}=\frac{1}{\alpha} \sum_{i=1}^{n} C \Gamma_{i} \Gamma_{i}^{*}=C\left(\frac{1}{\alpha} \sum_{i=1}^{n} P_{i}\right)=C$.

Consider an operator $\hat{C}: \hat{H} \rightarrow \hat{\tilde{H}}$ defined by

$$
\hat{C}=\frac{\alpha-1}{\alpha} \sum_{i=1}^{n} \tilde{\Delta}_{i} C_{i} \Delta_{i}^{*} .
$$

Using the following properties of the operators [7] $\left\{\Gamma_{i}\right\}_{i=1}^{n},\left\{\Gamma_{i}^{*}\right\}_{i=1}^{n},\left\{\Delta_{i}\right\}_{i=1}^{n},\left\{\Delta_{i}^{*}\right\}_{i=1}^{n}$ :

$$
\begin{aligned}
& \sum_{i=1}^{n} \Gamma_{i} \Delta_{i}^{*}=0 \\
& \Delta_{i}^{*} \Delta_{j}=-\frac{1}{\alpha-1} \Gamma_{i}^{*} \Gamma_{j}, \quad i \neq j,
\end{aligned}
$$

let us prove that

$$
\tilde{\Delta}_{k}^{*} \hat{C}=C_{k} \Delta_{k}^{*}, \quad k=1, \ldots, n .
$$

Indeed,

$$
\begin{aligned}
\tilde{\Delta}_{k}^{*} \hat{C} & =\tilde{\Delta}_{k}^{*}\left(\frac{\alpha-1}{\alpha} \sum_{i=1}^{n} \tilde{\Delta}_{i} C_{i} \Delta_{i}^{*}\right)=\frac{\alpha-1}{\alpha} \sum_{i=1}^{n}\left(\tilde{\Delta}_{k}^{*} \tilde{\Delta}_{i}\right) C_{i} \Delta_{i}^{*} \\
& =\frac{\alpha-1}{\alpha}\left(\tilde{\Delta}_{k}^{*} \tilde{\Delta}_{k}\right) C_{k} \Delta_{k}^{*}+\frac{\alpha-1}{\alpha} \sum_{\substack{i=1 \\
i \neq k}}^{n}\left(-\frac{1}{\alpha-1}\right) \tilde{\Gamma}_{k}^{*}\left(\tilde{\Gamma}_{i} C_{i}\right) \Delta_{i}^{*}
\end{aligned}
$$




$$
\begin{aligned}
& =\frac{\alpha-1}{\alpha} C_{k} \Delta_{k}^{*}-\frac{1}{\alpha} \sum_{\substack{i=1 \\
i \neq k}}^{n} \tilde{\Gamma}_{k}^{*}\left(\tilde{\Gamma}_{i} C_{i}\right) \Delta_{i}^{*}=\frac{\alpha-1}{\alpha} C_{k} \Delta_{k}^{*}-\frac{1}{\alpha} \tilde{\Gamma}_{k}^{*} C \sum_{\substack{i=1 \\
i \neq k}}^{n} \Gamma_{i} \Delta_{i}^{*} \\
& =\frac{\alpha-1}{\alpha} C_{k} \Delta_{k}^{*}-\frac{1}{\alpha} \tilde{\Gamma}_{k}^{*} C\left(\sum_{i=1}^{n} \Gamma_{i} \Delta_{i}^{*}-\Gamma_{k} \Delta_{k}^{*}\right)=\frac{\alpha-1}{\alpha} C_{k} \Delta_{k}^{*}+\frac{1}{\alpha}\left(\tilde{\Gamma}_{k}^{*}\right) C \Gamma_{k} \Delta_{k}^{*}=C_{k} \Delta_{k}^{*} .
\end{aligned}
$$

Now, let us show that

$$
C_{k}=\tilde{\Delta}_{k}^{*} \hat{C} \Delta_{k}, \quad k=1, \ldots, n .
$$

Using (2), (3), (7), (8), (9), and (11) we get

$$
\begin{aligned}
\tilde{\Delta}_{k}^{*} \hat{C} \Delta_{k} & =\tilde{\Delta}_{k}^{*}\left(\frac{\alpha-1}{\alpha} \sum_{i=1}^{n} \tilde{\Delta}_{i} C_{i} \Delta_{i}^{*}\right) \Delta_{k}=\frac{\alpha-1}{\alpha} \sum_{i=1}^{n} \tilde{\Delta}_{k}^{*} \tilde{\Delta}_{i} C_{i} \Delta_{i}^{*} \Delta_{k} \\
& =\frac{\alpha-1}{\alpha} \tilde{\Delta}_{k}^{*} \tilde{\Delta}_{k} C_{k} \Delta_{k}^{*} \Delta_{k}+\frac{\alpha-1}{\alpha} \sum_{\substack{i=1 \\
i \neq k}}^{n} \tilde{\Delta}_{k}^{*} \tilde{\Delta}_{i} C_{i} \Delta_{i}^{*} \Delta_{k} \\
& =\frac{\alpha-1}{\alpha} C_{k}+\frac{1}{\alpha(\alpha-1)} \sum_{\substack{i=1 \\
i \neq k}}^{n} \tilde{\Gamma}_{k}^{*} \tilde{\Gamma}_{i} C_{i} \Gamma_{i}^{*} \Gamma_{k} \\
& =\frac{\alpha-1}{\alpha} C_{k}+\frac{1}{\alpha-1} \tilde{\Gamma}_{k}^{*}\left(\frac{1}{\alpha} \sum_{\substack{i=1 \\
i \neq k}}^{n} \tilde{\Gamma}_{i} C_{i} \Gamma_{i}^{*}\right) \Gamma_{k} \\
& =\frac{\alpha-1}{\alpha} C_{k}+\frac{1}{\alpha-1} \tilde{\Gamma}_{k}^{*} C \Gamma_{k}-\frac{1}{\alpha(\alpha-1)} \tilde{\Gamma}_{k}^{*} \tilde{\Gamma}_{k} C_{k} \Gamma_{k}^{*} \Gamma_{k}=C_{k} .
\end{aligned}
$$

Now, it follows from (12) and (13) that $\tilde{Q}_{k} \hat{C}=\tilde{\Delta}_{k} \tilde{\Delta}_{k}^{*} \hat{C}=\tilde{\Delta}_{k} C_{k} \Delta_{k}^{*}=\tilde{Q}_{k} \hat{C} Q_{k}$, that is, $\tilde{Q}_{k} \hat{C}=\tilde{Q}_{k} \hat{C} Q_{k}, k=1, \ldots, n$. Whence,

$$
\hat{C}^{*} \tilde{Q}_{k}=Q_{k} \hat{C}^{*} \tilde{Q}_{k}, \quad k=1, \ldots, n .
$$

The latter identities mean that $\hat{C}^{*} \in \operatorname{Mor}\left(\mathcal{S}^{\prime}(\tilde{\pi}), \mathcal{S}^{\prime}(\pi)\right)$. The action of the auxiliary functors $\mathcal{T}^{\prime}$ and $\mathcal{S}^{\prime}$ on morphisms of the category Rep $\mathcal{P}_{n, \alpha}$ are defined by $\mathcal{T}^{\prime}(C)=C^{*}$ and $\mathcal{S}^{\prime}(C)=\hat{C}^{*}$ for any $C \in \operatorname{Mor}(\pi, \tilde{\pi})$. This completes the construction of the auxiliary functors.

Lemma 1. The functors $\mathcal{T}^{\prime}$ and $\mathcal{S}^{\prime}$ are category equivalences.

Proof. It is easy to check by using the definition that the functor $\mathcal{T}^{\prime}$ is univalent and complete. $\mathcal{T}^{2}=\operatorname{Id}$ and $\mathcal{T}^{\prime}(\pi)=\mathcal{T}(\pi)$ for any $\pi \in \operatorname{Rep} \mathcal{P}_{n, \alpha}$. Consequently, the functor $\mathcal{T}^{\prime}$ is an equivalence between the categories Rep $\mathcal{P}_{n, \alpha}$ and $\operatorname{Rep} \mathcal{P}_{n, n-\alpha}$.

Now, let us prove the lemma for the functor $\mathcal{S}^{\prime}$. Let us show that the functor $\mathcal{S}^{\prime}$ is univalent. Let $C, D \in \operatorname{Mor}(\pi, \tilde{\pi})$ and $C \neq D$, and show that $\mathcal{S}^{\prime}(C) \neq \mathcal{S}^{\prime}(D)$. Indeed, if $\mathcal{S}^{\prime}(C)=\mathcal{S}^{\prime}(D)$, then $\hat{C}^{*}=\hat{D}^{*}$ and $\hat{C}=\hat{D}$. By (13), we have

$$
C_{i}=\tilde{\Delta}_{i}^{*} \hat{C} \Delta_{i}=\tilde{\Delta}_{i}^{*} \hat{D} \Delta_{i}=D_{i}, \quad i=1, \ldots, n .
$$

Using the decomposition (8) we get

$$
C=\frac{1}{\alpha} \sum_{i=1}^{n} \tilde{\Gamma}_{i} C_{i} \Gamma_{i}^{*}, \quad D=\frac{1}{\alpha} \sum_{i=1}^{n} \tilde{\Gamma}_{i} D_{i} \Gamma_{i}^{*} .
$$

Then $C=D$ and, hence, the functor $\mathcal{S}^{\prime}$ is univalent. 
Let us now show that $\mathcal{S}^{\prime}$ is complete. Let $R \in \operatorname{Mor}\left(\mathcal{S}^{\prime}(\tilde{\pi}), \mathcal{S}^{\prime}(\pi)\right)$. To prove the completeness, construct a linear operator from the set $\operatorname{Mor}(\pi, \tilde{\pi})$ such that the functor takes on this morphism the value $R$. Since $R \in \operatorname{Mor}\left(\mathcal{S}^{\prime}(\tilde{\pi}), \mathcal{S}^{\prime}(\pi)\right)$, the operator $R: \hat{\tilde{H}} \rightarrow \hat{H}$ satisfies

$$
R \tilde{Q}_{k}=Q_{k} R \tilde{Q}_{k}, \quad k=1, \ldots, n .
$$

Consider an operator $\hat{r}$ in $B(\hat{H}, \hat{\tilde{H}})$ such that $\hat{r}^{*}=R$. Then the former identities can be written as

$$
\hat{r}^{*} \tilde{Q}_{k}=Q_{k} \hat{r}^{*} \tilde{Q}_{k}, \quad k=1, \ldots, n,
$$

and, consequently,

$$
\tilde{Q}_{k} \hat{r}=\tilde{Q}_{k} \hat{r} Q_{k}, \quad k=1, \ldots, n .
$$

Denote by $r_{k}$ the operators $r_{k}=\tilde{\Delta}_{k}^{*} \hat{r} \Delta_{k}: H_{k} \rightarrow \tilde{H}_{k}, k=1, \ldots, n$, and show that $\hat{r}$ can be represented as

$$
\hat{r}=\frac{\alpha-1}{\alpha} \sum_{k=1}^{n} \tilde{\Delta}_{k} r_{k} \Delta_{k}^{*}
$$

Indeed,

$$
\frac{\alpha-1}{\alpha} \sum_{k=1}^{n} \tilde{\Delta}_{k} r_{k} \Delta_{k}^{*}=\frac{\alpha-1}{\alpha} \sum_{k=1}^{n} \tilde{\Delta}_{k} \tilde{\Delta}_{k}^{*} \hat{r} \Delta_{k} \Delta_{k}^{*}=\frac{\alpha-1}{\alpha} \sum_{k=1}^{n} \tilde{Q}_{k} \hat{r} Q_{k}=\left(\frac{\alpha-1}{\alpha} \sum_{k=1}^{n} \tilde{Q}_{k}\right) \hat{r}=\hat{r} .
$$

It follows from the definition of $r_{k}$ and identities (3), (15) that

$$
\begin{aligned}
r_{k} \Delta_{k}^{*} & =\left(\tilde{\Delta}_{k}^{*} \hat{r} \Delta_{k}\right) \Delta_{k}^{*}=\tilde{\Delta}_{k}^{*} \hat{r}\left(\Delta_{k} \Delta_{k}^{*}\right)=\tilde{\Delta}_{k}^{*} \hat{r} Q_{k}=I_{\tilde{H}_{k}} \tilde{\Delta}_{k}^{*} \hat{r} Q_{k}=\left(\tilde{\Delta}_{k}^{*} \tilde{\Delta}_{k}\right) \tilde{\Delta}_{k}^{*} \hat{r} Q_{k} \\
& =\tilde{\Delta}_{k}^{*}\left(\tilde{\Delta}_{k} \tilde{\Delta}_{k}^{*}\right) \hat{r} Q_{k}=\tilde{\Delta}_{k}^{*} \tilde{Q}_{k} \hat{r} Q_{k}=\tilde{\Delta}_{k}^{*} \tilde{Q}_{k} \hat{r}=\tilde{\Delta}_{k}^{*}\left(\tilde{\Delta}_{k} \tilde{\Delta}_{k}^{*}\right) \hat{r}=\left(\tilde{\Delta}_{k}^{*} \tilde{\Delta}_{k}\right) \tilde{\Delta}_{k}^{*} \hat{r}=\tilde{\Delta}_{k}^{*} \hat{r} .
\end{aligned}
$$

Hence, we have

$$
r_{k} \Delta_{k}^{*}=\tilde{\Delta}_{k}^{*} \hat{r}, \quad k=1, \ldots, n .
$$

Consider the operator

$$
r=\frac{1}{\alpha} \sum_{i=1}^{n} \tilde{\Gamma}_{i} r_{i} \Gamma_{i}^{*}
$$

Using (2), (10), (11), (17) we get

$$
\begin{array}{ll}
r \Gamma_{k}=\tilde{\Gamma}_{k} r_{k}, & k=1, \ldots, n, \\
r_{k}=\tilde{\Gamma}_{k}^{*} r \Gamma_{k}, & k=1, \ldots, n .
\end{array}
$$

Indeed,

$$
\begin{aligned}
r \Gamma_{k} & =\frac{1}{\alpha} \sum_{i=1}^{n} \tilde{\Gamma}_{i} r_{i} \Gamma_{i}^{*} \Gamma_{k}=\frac{1}{\alpha} \tilde{\Gamma}_{k} r_{k}+\frac{1}{\alpha} \sum_{\substack{i=1 \\
i \neq j}}^{n} \tilde{\Gamma}_{i} r_{i}\left(\Gamma_{i}^{*} \Gamma_{k}\right)=\frac{1}{\alpha} \tilde{\Gamma}_{k} r_{k}-\frac{\alpha-1}{\alpha} \sum_{\substack{i=1 \\
i \neq j}}^{n} \tilde{\Gamma}_{i}\left(r_{i} \Delta_{i}^{*}\right) \Delta_{k} \\
& =\frac{1}{\alpha} \tilde{\Gamma}_{k} r_{k}-\frac{\alpha-1}{\alpha} \sum_{\substack{i=1 \\
i \neq j}}^{n} \tilde{\Gamma}_{i}\left(\tilde{\Delta}_{i}^{*} \hat{r}\right) \Delta_{k}=\frac{1}{\alpha} \tilde{\Gamma}_{k} r_{k}+\frac{\alpha-1}{\alpha} \tilde{\Gamma}_{k} \Delta_{k}^{*} \hat{r} \Delta_{k}=\tilde{\Gamma}_{k} r_{k}
\end{aligned}
$$


and

$$
\begin{aligned}
\tilde{\Gamma}_{k}^{*} r \Gamma_{k} & =\frac{1}{\alpha} \tilde{\Gamma}_{k}^{*}\left(\sum_{i=1}^{n} \tilde{\Gamma}_{i} r_{i} \Gamma_{i}^{*}\right) \Gamma_{k}=\frac{1}{\alpha} r_{k}+\frac{1}{\alpha} \sum_{\substack{i=1 \\
i \neq j}}^{n} \tilde{\Gamma}_{k}^{*} \tilde{\Gamma}_{i} r_{i} \Gamma_{i}^{*} \Gamma_{k} \\
& =\frac{1}{\alpha} r_{k}+\frac{(\alpha-1)^{2}}{\alpha} \sum_{\substack{i=1 \\
i \neq j}}^{n} \tilde{\Delta}_{k}^{*} \tilde{\Delta}_{i} r_{i} \Delta_{i}^{*} \Delta_{k}=\frac{1}{\alpha} r_{k}+(\alpha-1) \tilde{\Delta}_{k}^{*} \hat{r} \Delta_{k}-\frac{(\alpha-1)^{2}}{\alpha} r_{k}=r_{k} .
\end{aligned}
$$

It follows from (19) and (20) that $r P_{k}=r \Gamma_{k} \Gamma_{k}^{*}=\tilde{\Gamma}_{k} r_{k} \Gamma_{k}^{*}=\tilde{\Gamma}_{k} \tilde{\Gamma}_{k}^{*} r_{k} \Gamma_{k} \Gamma_{k}^{*}=\tilde{P}_{k} r P_{k}$, which means that $r \in \operatorname{Mor}(\pi, \tilde{\pi})$.

It is easy to check that $\mathcal{S}^{\prime}(r)=R$ and, consequently, the functor $\mathcal{S}^{\prime}$ is complete. So the univalence and completeness properties of the functor $\mathcal{S}^{\prime}$ are checked, $\mathcal{S}^{2}=\operatorname{Id}$ and $\mathcal{S}^{\prime}(\pi)=\mathcal{S}(\pi)$ for any $\pi \in \operatorname{Rep} \mathcal{P}_{n, \alpha}$. Consequently, the functor $\mathcal{S}^{\prime}$ is an equivalence between the categories $\operatorname{Rep} \mathcal{P}_{n, \alpha}$ and $\operatorname{Rep} \mathcal{P}_{n, \frac{\alpha}{\alpha-1}}$.

Lemma 2. If a system $S_{\pi}, \pi \in \operatorname{Rep} \mathcal{P}_{n, \mathrm{com}}$, of subspaces is transitive, then the system $S_{\Phi^{+}(\pi)}$

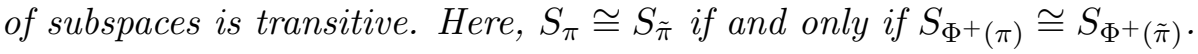

Proof. For the functors $\mathcal{T}$ and $\mathcal{S}$, we have $\mathcal{T}(\pi)=\mathcal{T}^{\prime}(\pi)$ and $\mathcal{S}(\pi)=\mathcal{S}^{\prime}(\pi)$ for any $\pi \in$ Rep $\mathcal{P}_{n, \alpha}$. Consequently, $S_{\mathcal{T}^{(\pi)}}=S_{\mathcal{T}^{\prime}(\pi)}$ and $S_{\mathcal{S}(\pi)}=S_{\mathcal{S}^{\prime}(\pi)}$. By Lemma 1, $\mathcal{T}^{\prime}$ is an equivalence of the categories that shows that if a system $S_{\pi}, \pi \in \operatorname{Rep} \mathcal{P}_{n, \text { com }}$, of subspaces is transitive, then the system $S_{\mathcal{T}(\pi)}$ of subspaces is transitive. We also have that $S_{\pi} \cong S_{\tilde{\pi}}$ if and only if $S_{\mathcal{T}(\pi)} \cong S_{\mathcal{T}(\tilde{\pi})}$.

Let us now consider the systems $S_{\mathcal{S}(\pi)}, \pi \in \operatorname{Rep} \mathcal{P}_{n, \alpha}$ of subspaces. Let $\pi, \tilde{\pi} \in \operatorname{Rep} \mathcal{P}_{n, \alpha}$. Consider the systems of subspaces $S_{\pi}=\left(H ; H_{1}, H_{2}, \ldots, H_{n}\right)$ and $S_{\tilde{\pi}}=\left(\tilde{H} ; \tilde{H}_{1}, \tilde{H}_{2}, \ldots, \tilde{H}_{n}\right)$, that, respectively, correspond to the representations $\pi$ and $\tilde{\pi}$. Let the systems of subspaces be isomorphic, that is, $S_{\pi} \cong S_{\tilde{\pi}}$. By the definition of isomorphic systems, there exists a linear operator $T \in B(H, \tilde{H})$ such that $T^{-1} \in B(\tilde{H}, H)$ and $T\left(H_{i}\right)=\tilde{H}_{i}, i=1, \ldots, n$. It follows from $T\left(H_{i}\right)=\tilde{H}_{i}, i=1, \ldots, n$, that $T\left(H_{i}\right) \subset \tilde{H}_{i}, i=1, \ldots, n$, and, consequently, we get the relations $T P_{i}=\tilde{P}_{i} T P_{i}, i=1, \ldots, n$. The latter relations mean that $T \in \operatorname{Mor}(\pi, \tilde{\pi})$ if $\hat{T}^{*} \in \operatorname{Mor}\left(\mathcal{S}^{\prime}(\tilde{\pi}), \mathcal{S}^{\prime}(\pi)\right)$, and

$$
\hat{T}^{*}\left(\operatorname{Im} \tilde{Q}_{i}\right) \subset\left(\operatorname{Im} Q_{i}\right), \quad i=1, \ldots, n .
$$

Again, using $T\left(H_{i}\right)=\tilde{H}_{i}, i=1, \ldots, n$, we get $T\left(H_{i}\right) \supset \tilde{H}_{i}, i=1, \ldots, n$, so that $T^{-1}\left(\tilde{H}_{i}\right) \subset$ $H_{i}, i=1, \ldots, n$, and, respectively, $T^{-1} \tilde{P}_{i}=P_{i} T^{-1} \tilde{P}_{i}, i=1, \ldots, n$. This means that $T^{-1} \in$ $\operatorname{Mor}(\tilde{\pi}, \pi)$, hence, ${\widehat{T^{-1}}}^{*} \in \operatorname{Mor}\left(\mathcal{S}^{\prime}(\pi), \mathcal{S}^{\prime}(\tilde{\pi})\right)$, and using ${\widehat{T^{-1}}}^{*}=\left(\hat{T}^{-1}\right)^{*}=\left(\hat{T}^{*}\right)^{-1}$ we get

$$
\operatorname{Im} \tilde{Q}_{i} \supset\left(\hat{T}^{*}\right)^{-1}\left(\operatorname{Im} Q_{i}\right), \quad i=1, \ldots, n,
$$

so that

$$
\hat{T}^{*}\left(\operatorname{Im} \tilde{Q}_{i}\right) \supset \operatorname{Im} Q_{i}, \quad i=1, \ldots, n .
$$

It follows from (21) and (22) that

$$
\hat{T}^{*}\left(\operatorname{Im} \tilde{Q}_{i}\right)=\operatorname{Im} Q_{i}, \quad i=1, \ldots, n,
$$

i.e., it is an isomorphism of the systems corresponding to the representations $\mathcal{S}^{\prime}(\pi)$ and $\mathcal{S}^{\prime}(\tilde{\pi})$ and, since the functors $\mathcal{S}^{\prime}$ and $\mathcal{S}$ coincide on the objects of the categories, it is an isomorphism of the systems corresponding to the representations $\mathcal{S}(\pi)$ and $\mathcal{S}(\tilde{\pi})$.

Since $\mathcal{S}^{\prime}$ is complete, using similar reasonings it is easy to show that the functor $\mathcal{S}^{\prime}$ and, hence, $\mathcal{S}$ takes the representations corresponding to nonisomorphic systems to representations that also correspond to nonisomorphic systems. 
Let again $\pi$ be a representation of the algebra $\mathcal{P}_{n, \alpha}$, and $\pi\left(p_{i}\right)=P_{i}, i=1, \ldots, n$, be orthogonal projections on a representation space $H$. Assume that the system of projections $P_{1}, P_{2}, \ldots, P_{n}$ gives rise to a transitive system of subspaces $S_{\pi}=\left(H ; H_{1}, H_{2}, \ldots, H_{n}\right)$, where $H_{i}=P_{i} H$, $i=1, \ldots, n$, that is,

$$
\operatorname{End}\left(S_{\pi}\right)=\left\{r \in B(H) \mid r\left(H_{i}\right) \subset H_{i}, i=1, \ldots, n\right\}=\operatorname{Mor}(\pi, \pi)=\mathbb{C} I .
$$

Consider $\mathcal{S}^{\prime}(\pi)=\hat{\pi}$, where $\hat{\pi}\left(q_{i}\right)=Q_{i}, i=1, \ldots, n$, and the corresponding system of subspaces $S_{\hat{\pi}}$. Let now $R \in \operatorname{End}\left(S_{\hat{\pi}}\right)$. Since $\operatorname{End}\left(S_{\hat{\pi}}\right)=\operatorname{Mor}\left(\mathcal{S}^{\prime}(\pi), \mathcal{S}^{\prime}(\pi)\right)$ and the functor $\mathcal{S}^{\prime}$ is complete, we see that $\mathcal{S}^{\prime}(r)=R$, where $r \in \operatorname{Mor}(\pi, \pi)$ is constructed from the operator $R^{*}=\frac{\alpha-1}{\alpha} \sum_{k=1}^{n} \tilde{\Delta}_{k} r_{k} \Delta_{k}^{*}, r_{i}=\tilde{\Delta}_{i}^{*} R^{*} \Delta_{i}: H_{i} \rightarrow \tilde{H}_{i}, i=1, \ldots, n$, as follows:

$$
r=\frac{1}{\alpha} \sum_{i=1}^{n} \Gamma_{i} r_{i} \Gamma_{i}^{*} .
$$

By using $R \in \operatorname{Mor}\left(\mathcal{S}^{\prime}(\pi), \mathcal{S}^{\prime}(\pi)\right)$, we obtain, similarly to (20), that

$$
r_{i}=\Gamma_{i}^{*} r \Gamma_{i}, \quad i=1, \ldots, n .
$$

Since the system $S_{\pi}$ is transitive, the operator $r$ is a scalar, that is, $r=\lambda I_{H}$. Using that $\Gamma_{i}^{*} \Gamma_{i}=I_{H_{i}}, i=1, \ldots, n$, and $(24)$ we get

$$
r_{i}=\lambda I_{H_{i}}, \quad i=1, \ldots, n
$$

Then $R^{*}$ is a scalar operator and, consequently, $R$ is also a scalar operator that means that the system $S_{\mathcal{S}^{\prime}(\pi)}$ is transitive and such is $S_{\mathcal{S}(\pi)}$.

The statement of Theorem 3 follows directly from Lemma 2.

\section{Transitive systems of subspaces corresponding to Rep $\mathcal{P}_{n, \text { abo, } \tau}$}

\subsection{Equivalence of the categories $\operatorname{Rep} \mathcal{P}_{n, \alpha}$ and $\operatorname{Rep} \mathcal{P}_{n, \text { abo, } \tau}$}

Let us examine the equivalence $\mathcal{F}$, constructed in [10], between the categories of $*$-representations $\mathcal{P}_{n, \alpha}$ and $\mathcal{P}_{n, \text { abo }, \frac{1}{\alpha}}, \alpha \neq 0$. Theorem 3 allows to consider nonisomorphic transitive systems of $n$ subspaces of the form $S_{\pi}$, constructed from representations of the algebras $\mathcal{P}_{n, \alpha}$ for $\alpha$ lying in the discrete spectrum. The equivalence $\mathcal{F}$, in its turn, allows to construct nonisomorphic transitive systems $S_{\mathcal{F}(\pi)}$ of $n+1$ subspaces starting with nonisomorphic transitive systems $S_{\pi}$, $\pi \in \mathcal{P}_{n, \alpha}$, of $n$ subspaces.

Let us describe the equivalence $\mathcal{F}$. Let $\pi$ be a representation of the algebra $\mathcal{P}_{n, \alpha}$, and $\pi\left(p_{i}\right)=$ $P_{i}, i=1, \ldots, n$, be orthogonal projections on a representation space $H$. As it was done in Section 4, let us introduce the spaces $H_{i}=\operatorname{Im} P_{i}$ and the natural isometries $\Gamma_{i}: H_{i} \rightarrow H$. Let $\mathcal{H}=H_{1} \oplus H_{2} \oplus \cdots \oplus H_{n}$. Define a linear operator $\Gamma: H_{1} \oplus H_{2} \oplus \cdots \oplus H_{n} \rightarrow H$ in terms of the matrix $\Gamma=\left(\Gamma_{1} \Gamma_{2} \ldots \Gamma_{n}\right)$ of the dimension $n \times 1$. Let $Q_{i}$ denote $n$ orthogonal projections, $Q_{i}=\operatorname{diag}\left(0, \ldots, 0, I_{H_{i}}, 0, \ldots, 0\right), i=1, \ldots, n$, and $P: \mathcal{H} \rightarrow \mathcal{H}$ an orthogonal projection defined by $P=\frac{1}{\alpha} \Gamma^{*} \Gamma$ with the block matrix $P=\frac{1}{\alpha}\left\|\Gamma_{i}^{*} \Gamma_{j}\right\|_{i, j=1}^{n}$ on the space $H_{1} \oplus H_{2} \oplus \cdots \oplus H_{n}$.

Let a functor $\mathcal{F}: \operatorname{Rep} \mathcal{P}_{n, \alpha} \rightarrow \operatorname{Rep} \mathcal{P}_{n \text {,abo, } \frac{1}{\alpha}}, \alpha \neq 0$, be defined on objects of the category of representations as follows: $\mathcal{F}(\pi)=\hat{\pi}$, where $\hat{\pi}\left(q_{i}\right)=Q_{i}, i=1, \ldots, n$, and $\hat{\pi}(p)=P$. The

identities $\sum_{i=1}^{n} Q_{i}=I$ and $Q_{i} P Q_{i}=\frac{1}{\alpha} Q_{i}, i=1, \ldots, n$, are easily checked. We do not describe the action of the functor $\mathcal{F}$ on morphisms of the category $\operatorname{Rep} \mathcal{P}_{n, \alpha}$, since we will not use it. 
Theorem 4. Systems of $n+1$ subspaces, $S_{\mathcal{F}(\pi)}$, constructed from irreducible inequivalent representations $\pi \in \operatorname{Rep} \mathcal{P}_{n, \alpha}$, where $\alpha$ is in the discrete spectrum, are nonisomorphic and transitive.

To prove the theorem, construct an auxiliary functor $\mathcal{F}^{\prime}: \operatorname{Rep} \mathcal{P}_{n, \alpha} \rightarrow \operatorname{Rep} \mathcal{P}_{n, \text { abo, } \frac{1}{\alpha}}, \alpha \neq 0$, the action of which on objects coincides with the action of $\mathcal{F}$, that is, $\mathcal{F}^{\prime}(\pi)=\mathcal{F}(\pi)$ for all $\pi \in$ $\operatorname{Rep} \mathcal{P}_{n, \alpha}$. Morphisms are defined as in Section 4. Let $\pi \in \operatorname{Rep}\left(\mathcal{P}_{n, \alpha}, H\right)$ and $\tilde{\pi} \in \operatorname{Rep}\left(\mathcal{P}_{n, \alpha}, \tilde{H}\right)$. A linear operator $C \in B(H, \tilde{H})$ will be called a morphism of the category of representations, written $C \in \operatorname{Mor}(\pi, \tilde{\pi})$, if $C \pi\left(p_{i}\right)=\tilde{\pi}\left(p_{i}\right) C \pi\left(p_{i}\right)$, that is,

$$
C P_{i}=\tilde{P}_{i} C P_{i}, \quad i=1, \ldots, n .
$$

As it was for the functors in Section 4 , denote the restrictions $\left.C\right|_{H_{i}}, i=1, \ldots, n$, by $C_{i}$. Then, as in Section 4, the operators $C_{i}$ map $H_{i}$ into $\tilde{H}_{i}$, that is,

$$
C_{i}\left(H_{i}\right) \subset \tilde{H}_{i}, \quad i=1, \ldots, n .
$$

It follows from $(25)$ and $(26)$ that

$$
C \Gamma_{i}=\tilde{\Gamma}_{i} C_{i}, \quad i=1, \ldots, n .
$$

The identities (25) are equivalent to the inclusions $C\left(H_{i}\right) \subset \tilde{H}_{i}, i=1, \ldots, n$, whence it follows that

$$
C_{i}=\tilde{\Gamma}_{i}^{*} C \Gamma_{i}, \quad i=1, \ldots, n .
$$

Similarly to Section 4, identities (27) allow to represent $C$ as

$$
C=\frac{1}{\alpha} \sum_{i=1}^{n} \tilde{\Gamma}_{i} C_{i} \Gamma_{i}^{*}
$$

The above presents all the similarities with the calculations performed in Section 4 ; the operator $\hat{C}$ is now defined differently. For the operator $\hat{C}=\operatorname{diag}\left(C_{1}, C_{2}, \ldots, C_{n}\right): \mathcal{H} \rightarrow \tilde{\mathcal{H}}$, it is easy to check that $\hat{C} Q_{i}=\tilde{Q}_{i} \hat{C}, i=1, \ldots, n$. Then $Q_{i} \hat{C}^{*}=\hat{C}^{*} \tilde{Q}_{i}, i=1, \ldots, n$. The latter allows to conclude that $\hat{C}^{*}\left(\operatorname{Im} \tilde{Q}_{i}\right) \subset \operatorname{Im} Q_{i}$ and, consequently,

$$
\hat{C}^{*} \tilde{Q}_{i}=Q_{i} \hat{C}^{*} \tilde{Q}_{i}, \quad i=1, \ldots, n .
$$

Denote by $(\tilde{P} \hat{C} P)_{i j}$ the elements of the block matrix of the operator $\tilde{P} \hat{C} P: H_{1} \oplus H_{2} \oplus \cdots \oplus$ $H_{n} \rightarrow \tilde{H}_{1} \oplus \tilde{H}_{2} \oplus \cdots \oplus \tilde{H}_{n}$. Then $(\tilde{P} \hat{C} P)_{i j}=\frac{1}{\alpha^{2}} \sum_{k=1}^{n} \tilde{\Gamma}_{i}^{*} \tilde{\Gamma}_{k} C_{k} \Gamma_{k}^{*} \Gamma_{j}=\frac{1}{\alpha^{2}} \tilde{\Gamma}_{i}^{*}\left(\sum_{k=1}^{n} \tilde{\Gamma}_{k} C_{k} \Gamma_{k}^{*}\right) \Gamma_{j}=$ $\frac{1}{\alpha} \tilde{\Gamma}_{i}^{*} C \Gamma_{j}=\frac{1}{\alpha} \tilde{\Gamma}_{i}^{*} \tilde{\Gamma}_{j} C_{j}=(\tilde{P} \hat{C})_{i j}$, that is, $\tilde{P} \hat{C} P=\tilde{P} \hat{C}$ and, consequently,

$$
\hat{C}^{*} \tilde{P}=P \hat{C}^{*} \tilde{P} \text {. }
$$

Identities (30) and (31) mean that $\hat{C}^{*} \in \operatorname{Mor}\left(\mathcal{F}^{\prime}(\tilde{\pi}), \mathcal{F}^{\prime}(\pi)\right)$. Define $\mathcal{F}^{\prime}(C)=\hat{C}^{*}$, and this finishes the construction of the functor $\mathcal{F}^{\prime}$.

Lemma 3. The functor $\mathcal{F}^{\prime}$ is an equivalence between the categories.

Proof. Let us show that the functor is univalent. Let $C, D \in \operatorname{Mor}(\pi, \tilde{\pi})$ and $C \neq D$, and show that $\mathcal{F}^{\prime}(C) \neq \mathcal{F}^{\prime}(D)$. Indeed, if $\mathcal{F}^{\prime}(C)=\mathcal{F}^{\prime}(D)$, i.e., $\hat{C}^{*}=\hat{D}^{*}$, then $C_{i}=D_{i}, \forall i=1, \ldots, n$. Let us use (29),

$$
C=\frac{1}{\alpha} \sum_{i=1}^{n} \tilde{\Gamma}_{i} C_{i} \Gamma_{i}^{*}, \quad D=\frac{1}{\alpha} \sum_{i=1}^{n} \tilde{\Gamma}_{i} D_{i} \Gamma_{i}^{*} .
$$


It follows from $C_{i}=D_{i}, i=1, \ldots, n$, and the form of the representation operators $C$ and $D$ that $C=D$ and, hence, the functor $\mathcal{F}^{\prime}$ is univalent.

Let us show that $\mathcal{F}^{\prime}$ is complete. Let $R \in \operatorname{Mor}\left(\mathcal{F}^{\prime}(\tilde{\pi}), \mathcal{F}^{\prime}(\pi)\right)$ and construct a linear operator in the set $\operatorname{Mor}(\pi, \tilde{\pi})$ such that the value of this functor on the morphism is $R$. It follows from $R \in \operatorname{Mor}\left(\mathcal{F}^{\prime}(\tilde{\pi}), \mathcal{F}^{\prime}(\pi)\right)$ that the operator $R: \tilde{\mathcal{H}} \rightarrow \mathcal{H}$ satisfies

$$
Q_{i} R \tilde{Q}_{i}=R \tilde{Q}_{i}, \quad i=1, \ldots, n, \quad P R \tilde{P}=R \tilde{P} .
$$

Denote by $\hat{r}$ an operator in $B(\mathcal{H}, \tilde{\mathcal{H}})$ such that $\hat{r}^{*}=R$. Then the latter identities can be rewritten as follows:

$$
Q_{i} \hat{r}^{*} \tilde{Q}_{i}=\hat{r}^{*} \tilde{Q}_{i}, \quad i=1, \ldots, n, \quad P \hat{r}^{*} \tilde{P}=\hat{r}^{*} \tilde{P},
$$

and, consequently,

$$
\tilde{Q}_{i} \hat{r} Q_{i}=\tilde{Q}_{i} \hat{r}, \quad i=1, \ldots, n
$$

and

$$
\tilde{P} \hat{r} P=\tilde{P} \hat{r}
$$

Let now $r_{i j}$ be elements of the block matrix of the operator $\hat{r}$ from $H_{1} \oplus H_{2} \oplus \cdots \oplus H_{n}$ into $\tilde{H}_{1} \oplus \tilde{H}_{2} \oplus \cdots \oplus \tilde{H}_{n}$. Identities (32) imply that if $i \neq j$, then $r_{i j}=0$. Denote $r_{i}=r_{i i}, i=1, \ldots, n$. Then $r_{i}: H_{i} \rightarrow \tilde{H}_{i}, i=1, \ldots, n$, and $\hat{r}=\operatorname{diag}\left(r_{1}, r_{2}, \ldots, r_{n}\right)$. Consider $r: H \rightarrow \tilde{H}$ defined by

$$
r=\frac{1}{\alpha} \tilde{\Gamma} \hat{r} \Gamma^{*} .
$$

Identity (33) and definition (34) imply that $\frac{1}{\alpha} \tilde{\Gamma}^{*} r \Gamma=\tilde{P} \hat{r} P=\tilde{P} \hat{r}$, then comparing the elements on the main diagonal of the corresponding block matrices gives

$$
r_{i}=\tilde{\Gamma}_{i}^{*} r \Gamma_{i}, \quad i=1, \ldots, n .
$$

Using the relation $\frac{1}{\alpha} \tilde{\Gamma} \tilde{\Gamma}^{*}=I_{\tilde{H}}$ we get $r \Gamma=I_{\tilde{H}} r \Gamma=\left(\frac{1}{\alpha} \tilde{\Gamma} \tilde{\Gamma}^{*}\right) r \Gamma=\tilde{\Gamma}\left(\frac{1}{\alpha} \tilde{\Gamma}^{*} r \Gamma\right)=\tilde{\Gamma}(\tilde{P} \hat{r} P)=$ $\tilde{\Gamma}(\tilde{P} \hat{r})=\tilde{\Gamma}\left(\frac{1}{\alpha} \tilde{\Gamma}^{*} \tilde{\Gamma}\right) \hat{r}=\left(\frac{1}{\alpha} \tilde{\Gamma} \tilde{\Gamma}^{*}\right) \tilde{\Gamma} \hat{r}=I_{\tilde{H}} \tilde{\Gamma} \hat{r}=\tilde{\Gamma} \hat{r}$. Rewrite the identity $r \Gamma=\tilde{\Gamma} \hat{r}$ in the matrix form,

$$
\left(r \Gamma_{1} r \Gamma_{2} \ldots r \Gamma_{n}\right)=\left(\tilde{\Gamma}_{1} r_{1} \tilde{\Gamma}_{2} r_{2} \ldots \tilde{\Gamma}_{n} r_{n}\right)
$$

that gives

$$
r \Gamma_{i}=\tilde{\Gamma}_{i} r_{i}, \quad i=1, \ldots, n .
$$

Using identities (35) and (36) we get

$$
r P_{i}=\tilde{P}_{i} r P_{i}, \quad i=1, \ldots, n .
$$

Indeed, $r P_{i}=r \Gamma_{i} \Gamma_{i}^{*}=\tilde{\Gamma}_{i} r_{i} \Gamma_{i}^{*}=\tilde{\Gamma}_{i} \tilde{\Gamma}_{i}^{*} r \Gamma_{i} \Gamma_{i}^{*}=\tilde{P}_{i} r P_{i}$. Identities (37) mean that $r \in \operatorname{Mor}(\pi, \tilde{\pi})$. Let us check that $\mathcal{F}^{\prime}(r)=\hat{r}^{*}=R$. Denote by $C$ the constructed morphism $r$ and find $\mathcal{F}^{\prime}(C)$. Since $\mathcal{F}^{\prime}(C)=\hat{C}^{*}$, where $\hat{C}=\operatorname{diag}\left(C_{1}, C_{2}, \ldots, C_{n}\right): \mathcal{H} \rightarrow \tilde{\mathcal{H}}$, let us find $C_{i}=\left.C\right|_{H_{i}}=\left.r\right|_{H_{i}}$, $i=1, \ldots, n$. Since $C \in \operatorname{Mor}(\pi, \tilde{\pi})$, it follows from (28) and (35) that $C_{i}=\tilde{\Gamma}_{i}^{*} C \Gamma_{i}=\tilde{\Gamma}_{i}^{*} r \Gamma_{i}=r_{i}$. Then $\hat{C}=\hat{r}, \hat{C}^{*}=\hat{r}^{*}$ and $\mathcal{F}^{\prime}(r)=\mathcal{F}^{\prime}(C)=\hat{C}^{*}=\hat{r}^{*}=R$. This proves that the functor $\mathcal{F}^{\prime}$ is complete.

Since $\mathcal{F}^{\prime}(\pi)=\mathcal{F}(\pi)$ for any $\pi \in \operatorname{Rep} \mathcal{P}_{n, \alpha}$ and $\mathcal{F}^{2}=\mathrm{Id}$, we see that $\mathcal{F}^{\prime}$ is an equivalence of the categories. 
Lemma 4. If a system $S_{\pi}, \pi \in \operatorname{Rep} \mathcal{P}_{n, \mathrm{com}}$, of $n$ subspaces is transitive, then the system $S_{\mathcal{F}(\pi)}$ of $n+1$ subspaces is transitive. Also, $S_{\pi} \cong S_{\tilde{\pi}}$ if and only if $S_{\mathcal{F}(\pi)} \cong S_{\mathcal{F}(\tilde{\pi})}$.

Proof. Since the functors $\mathcal{F}$ and $\mathcal{F}^{\prime}$ coincide on the objects of the categories, the representations constructed using the functors and the corresponding systems of subspaces will coincide, $S_{\mathcal{F}(\pi)}=$ $S_{\mathcal{F}^{\prime}(\pi)}$ for $\forall \pi \in \operatorname{Rep} \mathcal{P}_{n, \alpha}$. Let $\pi, \tilde{\pi} \in \operatorname{Rep} \mathcal{P}_{n, \alpha}, \alpha \neq 0$, and the systems of subspaces $S_{\pi}=$ $\left(H ; H_{1}, H_{2}, \ldots, H_{n}\right)$ and $S_{\tilde{\pi}}=\left(\tilde{H} ; \tilde{H}_{1}, \tilde{H}_{2}, \ldots, \tilde{H}_{n}\right)$, which correspond to the representations $\pi$ and $\tilde{\pi}$, be isomorphic, that is, $S_{\pi} \cong S_{\tilde{\pi}}$. By the definition of isomorphic systems, there exists a linear operator $T \in B(H, \tilde{H})$ such that $T^{-1} \in B(\tilde{H}, H)$ and $T\left(H_{i}\right)=\tilde{H}_{i}, i=1, \ldots, n$. It follows from $T\left(H_{i}\right)=\tilde{H}_{i}, i=1, \ldots, n$, that $T\left(H_{i}\right) \subset \tilde{H}_{i}, i=1, \ldots, n$, and, consequently, $T P_{i}=\tilde{P}_{i} T P_{i}$, $i=1, \ldots, n$. The latter identities mean that $T \in \operatorname{Mor}(\pi, \tilde{\pi})$. Then $\hat{T}^{*} \in \operatorname{Mor}\left(\mathcal{F}^{\prime}(\tilde{\pi}), \mathcal{F}^{\prime}(\pi)\right)$ and

$$
\hat{T}^{*}\left(\operatorname{Im} \tilde{Q}_{i}\right) \subset\left(\operatorname{Im} Q_{i}\right)(i=1, \ldots, n) \quad \text { and } \quad \hat{T}^{*}(\operatorname{Im} \tilde{P}) \subset(\operatorname{Im} P) .
$$

Again, considering the identities $T\left(H_{i}\right)=\tilde{H}_{i}, i=1, \ldots, n$, we conclude that $T\left(H_{i}\right) \supset \tilde{H}_{i}, i=$ $1, \ldots, n$, that is, $T^{-1}\left(\tilde{H}_{i}\right) \subset H_{i}, i=1, \ldots, n$, and, respectively, $T^{-1} \tilde{P}_{i}=P_{i} T^{-1} \tilde{P}_{i}, i=1, \ldots, n$. These identities imply that $T^{-1} \in \operatorname{Mor}(\tilde{\pi}, \pi)$. Then $\widehat{T}^{-1}{ }^{*} \in \operatorname{Mor}\left(\mathcal{F}^{\prime}(\pi), \mathcal{F}^{\prime}(\tilde{\pi})\right)$, whence using $\widehat{T}^{-1}{ }^{*}=\left(\hat{T}^{-1}\right)^{*}=\left(\hat{T}^{*}\right)^{-1}$ we have

$$
\operatorname{Im} \tilde{Q}_{i} \supset\left(\hat{T}^{*}\right)^{-1}\left(\operatorname{Im} Q_{i}\right), \quad i=1, \ldots, n \quad \text { and } \quad \operatorname{Im} \tilde{P} \supset\left(\hat{T}^{*}\right)^{-1}(\operatorname{Im} P),
$$

and, consequently,

$$
\hat{T}^{*}\left(\operatorname{Im} \tilde{Q}_{i}\right) \supset \operatorname{Im} Q_{i}, \quad i=1, \ldots, n \quad \text { and } \quad \hat{T}^{*}(\operatorname{Im} \tilde{P}) \supset \operatorname{Im} P .
$$

It follows from (38) and (39) that

$$
\hat{T}^{*}\left(\operatorname{Im} \tilde{Q}_{i}\right)=\operatorname{Im} Q_{i}, \quad i=1, \ldots, n \quad \text { and } \quad \hat{T}^{*}(\operatorname{Im} \tilde{P})=\operatorname{Im} P
$$

that shows that it is an isomorphism of the systems that correspond to the representations $\mathcal{F}^{\prime}(\pi)$ and $\mathcal{F}^{\prime}(\tilde{\pi})$ and, since the functors $\mathcal{F}^{\prime}$ and $\mathcal{F}$ coincide on the objects of the category, it is an isomorphism of the systems corresponding to the representations $\mathcal{F}(\pi)$ and $\mathcal{F}(\tilde{\pi})$.

Since the functor $\mathcal{F}^{\prime}$ is complete, similar reasonings show that the representations that correspond to nonisomorphic systems are mapped by the functor $\mathcal{F}^{\prime}$, and hence the functor $\mathcal{F}$, into representations that give rise to nonisomorphic systems.

Let us now prove the first part of the proposition. Let $\pi$ be a representation of the algebra $\mathcal{P}_{n, \alpha}$ and $\pi\left(p_{i}\right)=P_{i}, i=1, \ldots, n$, be orthogonal projections on a representation space $H$. And let the system of orthogonal projections $P_{1}, P_{2}, \ldots, P_{n}$ induce a transitive system of subspaces $S_{\pi}=\left(H ; H_{1}, H_{2}, \ldots, H_{n}\right)$, where $H_{i}=P_{i} H, i=1, \ldots, n$, that is,

$$
\operatorname{End}\left(S_{\pi}\right)=\left\{r \in B(H) \mid r\left(H_{i}\right) \subset H_{i}, i=1, \ldots, n\right\}=\operatorname{Mor}(\pi, \pi)=\mathbb{C} I .
$$

Consider $\mathcal{F}^{\prime}(\pi)=\hat{\pi}$, where $\hat{\pi}\left(q_{i}\right)=Q_{i}, i=1, \ldots, n$, and $\hat{\pi}(p)=P$, and the corresponding system $S_{\hat{\pi}}$ of subspaces. Let now $R \in \operatorname{End}\left(S_{\hat{\pi}}\right)$. Using $\operatorname{End}\left(S_{\hat{\pi}}\right)=\operatorname{Mor}\left(\mathcal{F}^{\prime}(\pi), \mathcal{F}^{\prime}(\pi)\right)$ and since the functor $\mathcal{F}^{\prime}$ is complete, we see that $\mathcal{F}^{\prime}(r)=R$, where $r \in \operatorname{Mor}(\pi, \pi)$ is constructed from the diagonal operator $R^{*}=\operatorname{diag}\left(r_{1}, r_{2}, \ldots, r_{n}\right)$ on the space $H_{1} \oplus H_{2} \oplus \cdots \oplus H_{n}$ as follows:

$$
r=\frac{1}{\alpha} \Gamma R^{*} \Gamma^{*}
$$

Using the inclusion $R \in \operatorname{Mor}\left(\mathcal{F}^{\prime}(\pi), \mathcal{F}^{\prime}(\pi)\right.$ ), which is similar to identity (35), we get

$$
r_{i}=\Gamma_{i}^{*} r \Gamma_{i}, \quad i=1, \ldots, n .
$$


Since the system $S_{\pi}$ is transitive, the operator $r$ is scalar, that is, $r=\lambda I_{H}$. Using $\Gamma_{i}^{*} \Gamma_{i}=I_{H_{i}}$, $i=1, \ldots, n$, and identities (41) we get

$$
r_{i}=\lambda I_{H_{i}}, \quad i=1, \ldots, n
$$

Then $R^{*}$ is a scalar operator and, consequently, $R$ is also a scalar operator that means that the system $S_{\mathcal{F}^{\prime}(\pi)}$ is transitive and such is $S_{\mathcal{F}(\pi)}$.

The claim of Theorem 4 follows from Theorem 3 and Lemma 4.

\subsection{Transitive quintuples of subspaces}

By Theorem 4, the functor $\mathcal{F}$ maps known nonisomorphic transitive quadruples of subspaces of the form $S_{\pi}$, where $\pi \in \operatorname{Rep} \mathcal{P}_{4 \text {,com }}$, into nonisomorphic transitive quintuples $S_{\mathcal{F}(\pi)}[11,12]$. In this section, we give inequivalent irreducible $*$-representations of the $*$-algebras $\mathcal{P}_{4, \text { abo }, \tau}, \tau \in \tilde{\Sigma}_{4}$, where $\tilde{\Sigma}_{4}$ that is the set of $\tau \in \mathbb{R}_{+}$such that there exists at least one $*$-representation of the $*$-algebra $\mathcal{P}_{4, \text { abo }, \tau}$, is related to $\Sigma_{4}$, the set $\alpha \in \mathbb{R}_{+}$such that there exists at least one *-representation of the $*$-algebra $\mathcal{P}_{4, \alpha}$, via the following relation [10]:

$$
\tilde{\Sigma}_{4}=\{0\} \cup\left\{\frac{1}{\alpha} \mid \alpha \neq 0, \alpha \in \Sigma_{4}\right\}
$$

Here, by [7], $\Sigma_{4}=\left\{0,1,2-\frac{2}{2 k+1}(k=1,2, \ldots), 2-\frac{1}{n}(n=2,3, \ldots), 2,2+\frac{1}{n}(n=2,3, \ldots), 2+\right.$ $\left.\frac{2}{2 k+1}(k=1,2, \ldots), 3,4\right\}$. For these representations, the corresponding systems of subspaces are nonisomorphic and transitive.

Let $e_{i, j}^{r \times s}$ denote an $(r \times s)$-matrix that has 1 at the intersection of the $i$ th row and the $j$ th column, with other elements being zero.

1) The $*$-algebra $\mathcal{P}_{4, \text { abo, } 0}$ has 4 irreducible inequivalent one-dimensional representations, $Q_{1}=$ $\cdots=Q_{k-1}=Q_{k+1}=\cdots=Q_{4}=P=0, Q_{k}=1$.

2) The *-algebra $\mathcal{P}_{4, \text { abo, } 1}$ has 4 irreducible inequivalent one-dimensional representations, $Q_{1}=$ $\cdots=Q_{k-1}=Q_{k+1}=\cdots=Q_{4}=0, P=Q_{k}=1$.

3) The *-algebra $\mathcal{P}_{4, \text { abo }, \frac{1}{3}}$ has 4 irreducible inequivalent three-dimensional representations that are unitary equivalent, up to a permutation, to

$$
\begin{array}{lll}
Q_{1}=1 \oplus 0 \oplus 0, & Q_{3}=0 \oplus 0 \oplus 1, & P=\frac{1}{3} \sum_{i, j=1}^{3} e_{i, j}^{3 \times 3}, \\
Q_{2}=0 \oplus 1 \oplus 0, & Q_{4}=0 \oplus 0 \oplus 0, & \mathcal{H}=\mathbb{C} \oplus \mathbb{C} \oplus \mathbb{C} .
\end{array}
$$

4) The *-algebra $\mathcal{P}_{4, \text { abo, } \frac{1}{4}}$ has a unique irreducible four dimensional representation,

$$
\begin{array}{rlrl}
Q_{1} & =1 \oplus 0 \oplus 0 \oplus 0, & Q_{3}=0 \oplus 0 \oplus 1 \oplus 0, & P=\frac{1}{4} \sum_{i, j=1}^{4} e_{i, j}^{4 \times 4}, \\
Q_{2}=0 \oplus 1 \oplus 0 \oplus 0, & Q_{4}=0 \oplus 0 \oplus 0 \oplus 1, & \mathcal{H}=\mathbb{C} \oplus \mathbb{C} \oplus \mathbb{C} \oplus \mathbb{C} .
\end{array}
$$

5) The $*$-algebra $\mathcal{P}_{4, \text { abo, } \frac{1}{2}}$ has 6 irreducible two-dimensional representations that are unitary equivalent, up to a permutation, to

$$
Q_{1}=1 \oplus 0, \quad Q_{2}=0 \oplus 1, \quad Q_{3}=0 \oplus 0, \quad Q_{4}=0 \oplus 0, \quad P=\frac{1}{2}\left(\begin{array}{ll}
1 & 1 \\
1 & 1
\end{array}\right)
$$


where the representation space is $\mathcal{H}=\mathbb{C} \oplus \mathbb{C}$, and the following inequivalent four dimensional representations that depend on the points of the set $\Omega=\left\{(a, b, c) \in \mathbb{R} \mid a^{2}+b^{2}+c^{2}=1, a>0\right.$, $b>0, c \in(-1,1)$; or $a=0, b^{2}+c^{2}=1, b>0, c>0$; or $\left.b=0, a^{2}+c^{2}=1, a>0, c>0\right\}$ :

$$
\begin{aligned}
& Q_{1}=1 \oplus 0 \oplus 0 \oplus 0, \quad Q_{3}=0 \oplus 0 \oplus 1 \oplus 0, \\
& Q_{2}=0 \oplus 1 \oplus 0 \oplus 0, \quad Q_{4}=0 \oplus 0 \oplus 0 \oplus 1 \text {, } \\
& P=\frac{1}{2}\left(\begin{array}{cccc}
1 & \frac{c(c-i b)}{\sqrt{1-a^{2}}} & \frac{b(b+i c)}{\sqrt{1-a^{2}}} & a \\
\frac{c(c+i b)}{\sqrt{1-a^{2}}} & 1 & -a & \frac{b(b-i c)}{\sqrt{1-a^{2}}} \\
\frac{b(b-i c)}{\sqrt{1-a^{2}}} & -a & 1 & \frac{c(c+i b)}{\sqrt{1-a^{2}}} \\
a & \frac{b(b+i c)}{\sqrt{1-a^{2}}} & \frac{c(c-i b)}{\sqrt{1-a^{2}}} & 1
\end{array}\right),
\end{aligned}
$$

where the representation space is $\mathcal{H}=\mathbb{C} \oplus \mathbb{C} \oplus \mathbb{C} \oplus \mathbb{C}$.

6) The $*$-algebras $\mathcal{P}_{4, \text { abo, } \frac{1}{\alpha}}$, for $\alpha=2-\frac{2}{2 k+1}, k=1,2, \ldots$, have unique irreducible representations

$$
\begin{aligned}
& Q_{1}=I \oplus 0 \oplus 0 \oplus 0, \quad Q_{3}=0 \oplus 0 \oplus I \oplus 0, \\
& Q_{2}=0 \oplus I \oplus 0 \oplus 0, \quad Q_{4}=0 \oplus 0 \oplus 0 \oplus I, \\
& P=\frac{1}{\alpha}\left(\begin{array}{cc}
A & B \\
B^{t} & C
\end{array}\right), \quad \text { where } \quad A=\left(\begin{array}{cc}
I & A_{1} \\
A_{1} & I
\end{array}\right), \quad C=\left(\begin{array}{cc}
I & C_{1} \\
C_{1} & I
\end{array}\right), \quad B=\left(\begin{array}{ll}
B_{00} & B_{01} \\
B_{10} & B_{11}
\end{array}\right), \\
& A_{1}=\frac{1}{2 k+1} \sum_{i=1}^{k}(2 k+3-4 i) e_{i, i}^{k \times k}, \quad C_{1}=\frac{1}{2 k+1} \sum_{i=1}^{k}(2 k+1-4 i) e_{i, i}^{k \times k}, \\
& B_{l m}=\frac{(-1)^{\ell}}{2 k+1} \sum_{i=1}^{k} \sqrt{(2 k-2 i+1)(2 i-1)} e_{i, i}^{k \times k}+\frac{(-1)^{m}}{2 k+1} \sum_{i=1}^{k-1} \sqrt{(2 k-2 i) 2 i} e_{i+1, i}^{k \times k},
\end{aligned}
$$

and the representation space is

$$
\mathcal{H}=\mathbb{C}^{k} \oplus \mathbb{C}^{k} \oplus \mathbb{C}^{k} \oplus \mathbb{C}^{k}
$$

7) The $*$-algebras $\mathcal{P}_{4, \text { abo, } \frac{1}{\alpha}}$, for $\alpha=2-\frac{1}{2 k+1}, k=1,2, \ldots$, have unique irreducible representations

$$
\begin{aligned}
& Q_{1}=I \oplus 0 \oplus 0 \oplus 0, \quad Q_{3}=0 \oplus 0 \oplus I \oplus 0, \\
& Q_{2}=0 \oplus I \oplus 0 \oplus 0, \quad Q_{4}=0 \oplus 0 \oplus 0 \oplus I, \\
& P=\frac{1}{\alpha}\left(\begin{array}{cc}
A & B \\
B^{t} & C
\end{array}\right), \quad \text { where } \quad A=\left(\begin{array}{cc}
I & A_{1} \\
A_{1}^{t} & I
\end{array}\right), \quad C=\left(\begin{array}{cc}
I & C_{1} \\
C_{1} & I
\end{array}\right), \quad B=\left(\begin{array}{cc}
\eta & \eta \\
B_{00} & B_{10} \\
B_{01} & B_{11}
\end{array}\right), \\
& \eta=(\sqrt{\frac{k}{2 k+1}}, \underbrace{0,0, \ldots, 0}_{k-1}), \\
& A_{1}=-\frac{1}{2 k+1} \sum_{i=1}^{k} 2 i e_{i+1, i}^{(k+1) \times k}, \quad C_{1}=-\frac{1}{2 k+1} \sum_{i=1}^{k}(2 i-1) e_{i, i}^{k \times k}, \\
& B_{l m}=\frac{(-1)^{\ell}}{4 k+2} \sum_{i=1}^{k} \sqrt{(2 k-2 i+1)(2 k+2 i)} e_{i, i}^{k \times k}+\frac{(-1)^{m}}{4 k+2} \sum_{i=1}^{k-1} \sqrt{(2 k-2 i)(2 k+2 i+1)} e_{i, i+1}^{k \times k},
\end{aligned}
$$

and the representation space is

$$
\mathcal{H}=\mathbb{C}^{k+1} \oplus \mathbb{C}^{k} \oplus \mathbb{C}^{k} \oplus \mathbb{C}^{k}
$$


8) The $*$-algebras $\mathcal{P}_{4, \text { abo }, \frac{1}{\alpha}}$, for $\alpha=2-\frac{1}{2 k}, k=1,2, \ldots$, have unique irreducible representations

$$
\begin{aligned}
& Q_{1}= I \oplus 0 \oplus 0 \oplus 0, \quad Q_{3}=0 \oplus 0 \oplus I \oplus 0, \\
& Q_{2}= 0 \oplus I \oplus 0 \oplus 0, \quad Q_{4}=0 \oplus 0 \oplus 0 \oplus I, \\
& P= \frac{1}{\alpha}\left(\begin{array}{cc}
A & B \\
B^{t} & C
\end{array}\right), \quad \text { where } \quad A=\left(\begin{array}{cc}
I & A_{1} \\
A_{1}^{t} & I
\end{array}\right), \quad C=\left(\begin{array}{cc}
I & C_{1} \\
C_{1} & I
\end{array}\right), \quad B=\left(\begin{array}{cc}
B_{00} & B_{10} \\
\eta & \eta \\
B_{01} & B_{11}
\end{array}\right), \\
& \eta=(\sqrt{\frac{2 k-1}{4 k}}, \underbrace{0,0, \ldots, 0}_{k-1}), \\
& A_{1}=-\frac{1}{k} \sum_{i=1}^{k-1} i e_{i, i+1}^{(k-1) \times k}, \quad C_{1}=-\frac{1}{2 k} \sum_{i=1}^{k}(2 i-1) e_{i, i}^{k \times k}, \\
& B_{l m}=\frac{(-1)^{\ell}}{4 k} \sum_{i=1}^{k-1} \sqrt{(2 k-2 i)(2 k+2 i-1)} e_{i, i}^{(k-1) \times k} \\
& \quad+\frac{(-1)^{m}}{4 k} \sum_{i=1}^{k-1} \sqrt{(2 k-2 i-1)(2 k+2 i)} e_{i, i+1}^{(k-1) \times k},
\end{aligned}
$$

and the representation space is

$$
\mathcal{H}=\mathbb{C}^{k-1} \oplus \mathbb{C}^{k} \oplus \mathbb{C}^{k} \oplus \mathbb{C}^{k}
$$

9) The $*$-algebras $\mathcal{P}_{4, \text { abo, } \frac{1}{\alpha}}$, for $\alpha=2+\frac{1}{2 k}, k=1,2, \ldots$, have unique irreducible representations

$$
\begin{aligned}
& Q_{1}= I \oplus 0 \oplus 0 \oplus 0, \quad Q_{3}=0 \oplus 0 \oplus I \oplus 0, \\
& Q_{2}= 0 \oplus I \oplus 0 \oplus 0, \quad Q_{4}=0 \oplus 0 \oplus 0 \oplus I, \\
& P= \frac{1}{\alpha}\left(\begin{array}{cc}
A & B \\
B^{t} & C
\end{array}\right), \quad \text { where } \quad A=\left(\begin{array}{cc}
I & A_{1} \\
A_{1}^{t} & I
\end{array}\right), \quad C=\left(\begin{array}{cc}
I & C_{1} \\
C_{1} & I
\end{array}\right), \quad B=\left(\begin{array}{cc}
\eta & \eta \\
B_{11} & B_{01} \\
B_{10} & B_{00}
\end{array}\right), \\
& \eta=(\sqrt{\frac{2 k+1}{4 k}}, \underbrace{0,0, \ldots, 0}_{k-1}), \\
& A_{1}=\frac{1}{k} \sum_{i=1}^{k} i e_{i+1, i}^{(k+1) \times k}, \quad C_{1}=\frac{1}{2 k} \sum_{i=1}^{k}(2 i-1) e_{i, i}^{k \times k}, \\
& B_{l m}=\frac{(-1)^{\ell}}{4 k} \sum_{i=1}^{k} \sqrt{(2 k+2 i)(2 k-2 i+1)} e_{i, i}^{k \times k} \\
& \quad+\frac{(-1)^{m}}{4 k} \sum_{i=1}^{k-1} \sqrt{(2 k+2 i+1)(2 k-2 i)} e_{i, i+1}^{k \times k},
\end{aligned}
$$

and the representation space is

$$
\mathcal{H}=\mathbb{C}^{k+1} \oplus \mathbb{C}^{k} \oplus \mathbb{C}^{k} \oplus \mathbb{C}^{k}
$$

10) The $*$-algebras $\mathcal{P}_{4, \text { abo }, \frac{1}{\alpha}}$, for $\alpha=2+\frac{1}{2 k+1}, k=1,2, \ldots$, have unique irreducible representations

$$
\begin{array}{ll}
Q_{1}=I \oplus 0 \oplus 0 \oplus 0, & Q_{3}=0 \oplus 0 \oplus I \oplus 0, \\
Q_{2}=0 \oplus I \oplus 0 \oplus 0, & Q_{4}=0 \oplus 0 \oplus 0 \oplus I,
\end{array}
$$




$$
\begin{aligned}
& P= \frac{1}{\alpha}\left(\begin{array}{cc}
A & B \\
B^{t} & C
\end{array}\right), \quad \text { where } A=\left(\begin{array}{cc}
I & A_{1} \\
A_{1}^{t} & I
\end{array}\right), \quad C=\left(\begin{array}{cc}
I & C_{1} \\
C_{1} & I
\end{array}\right), \quad B=\left(\begin{array}{cc}
B_{11} & B_{01} \\
\eta & \eta \\
B_{10} & B_{00}
\end{array}\right), \\
& \eta=(\sqrt{\frac{k+1}{2 k+1}}, \underbrace{0,0, \ldots, 0}_{k}), \\
& A_{1}=\frac{1}{2 k+1} \sum_{i=1}^{k} 2 i e_{i, i+1}^{k \times(k+1)}, \quad C_{1}=\frac{1}{2 k+1} \sum_{i=1}^{k+1}(2 i-1) e_{i, i}^{(k+1) \times(k+1)}, \\
& B_{l m}=\frac{(-1)^{\ell}}{4 k+2} \sum_{i=1}^{k} \sqrt{(2 k-2 i+2)(2 k+2 i+1)} e_{i, i}^{k \times(k+1)} \\
& \quad+\frac{(-1)^{m}}{4 k+2} \sum_{i=1}^{k} \sqrt{(2 k+2 i-1)(2 k+2 i+2)} e_{i, i+1}^{k \times(k+1)},
\end{aligned}
$$

and the representation space is

$$
\mathcal{H}=\mathbb{C}^{k} \oplus \mathbb{C}^{k+1} \oplus \mathbb{C}^{k+1} \oplus \mathbb{C}^{k+1}
$$

11) The $*$-algebras $\mathcal{P}_{4, \text { abo }, \frac{1}{\alpha}}$, for $\alpha=2+\frac{2}{2 k+1}, k=1,2, \ldots$, have unique irreducible representations

$$
\begin{aligned}
& Q_{1}=I \oplus 0 \oplus 0 \oplus 0, \quad Q_{3}=0 \oplus 0 \oplus I \oplus 0, \\
& Q_{2}=0 \oplus I \oplus 0 \oplus 0, \quad Q_{4}=0 \oplus 0 \oplus 0 \oplus I, \\
& P=\frac{1}{\alpha}\left(\begin{array}{cc}
A & B \\
B^{t} & C
\end{array}\right), \quad \text { where } \quad A=\left(\begin{array}{cc}
I & A_{1} \\
A_{1} & I
\end{array}\right), \quad C=\left(\begin{array}{cc}
I & C_{1} \\
C_{1} & I
\end{array}\right), \quad B=\left(\begin{array}{ll}
B_{11} & B_{01} \\
B_{10} & B_{00}
\end{array}\right), \\
& A_{1}=-\frac{1}{2 k+1} \sum_{i=1}^{k+1}(2 k+3-4 i) e_{i, i}^{(k+1) \times(k+1)}, \\
& C_{1}=e_{1,1}^{(k+1) \times(k+1)}-\frac{1}{2 k+1} \sum_{i=2}^{k+1}(2 k+5-4 i) e_{i, i}^{(k+1) \times(k+1)}, \\
& B_{l m}=\frac{1}{\sqrt{2 k+1}} e_{1,1}^{(k+1) \times(k+1)}+\frac{(-1)^{\ell}}{2 k+1} \sum_{i=2}^{k+1} \sqrt{(2 k-2 i+3)(2 i-1)} e_{i, i}^{(k+1) \times(k+1)} \\
& \quad+\frac{(-1)^{m}}{2 k+1} \sum_{i=1}^{k} \sqrt{(2 k-2 i+2) 2 i} e_{i, i+1}^{(k+1) \times(k+1)},
\end{aligned}
$$

and the representation space is

$$
\mathcal{H}=\mathbb{C}^{k+1} \oplus \mathbb{C}^{k+1} \oplus \mathbb{C}^{k+1} \oplus \mathbb{C}^{k+1} .
$$

\section{Acknowledgments}

The authors are grateful to S.A. Kruglyak for useful remarks and suggestions.

[1] Halmos P.R., Two subspaces, Trans. Amer. Math. Soc., 1969, V.144, 381-389.

[2] Halmos P.R., Ten problems in Hilbert space, Bull. Amer. Math. Soc., 1970, V.76, 887-933.

[3] Brenner S., Endomorphism algebras of vector spaces with distinguished sets of subspaces, J. Algebra, 1967, V.6, 100-114.

[4] Gel'fand I.M., Ponomarev V.A., Problems of linear algebra and classification of quadruples of subspaces in a finite-dimensional vector space, Coll. Math. Spc. Bolyai 5, Tihany, 1970, 163-237. 
[5] Nazarova L.A., Representations of a quadruple, Izv. AN SSSR, 1967, V.31, N 6, 1361-1377 (in Russian).

[6] Enomoto M., Watatani Ya., Relative position of four subspaces in a Hilbert space, math.OA/0404545.

[7] Kruglyak S.A., Rabanovich V.I., Samoǐlenko Yu.S., On sums of projections, Funktsional. Anal. i Prilozhen., 2002, V.36, N 3, 30-35 (English transl.: Funct. Anal. Appl., 2002, V.36, N 3, 182-195).

[8] Kruglyak S.A., Samoľlenko Yu.S., On the complexity of description of representations of *-algebras generated by idempotents, Proc. Amer. Math. Soc., 2000, V.128, 1655-1664.

[9] Ostrovskyi V.L., Samoǐlenko Yu.S., Introduction to the theory of representations of finitely presented *-algebras. I. Representations by bounded operators, Harwood Acad. Publs., 1999.

[10] Popovich S.V., Samořlenko Yu.S., On homomorphisms of algebras generated by projections, and the Coxeter functors, Ukrain. Mat. Zh., 2003, V.55, N 9, 1224-1237 (English transl.: Ukrainian Math. J., 2003, V.55, N 9, 1480-1496).

[11] Moskaleva Yu.P., Samoǐlenko Yu.S., Systems of $n$ subspaces and representations of *-algebras generated by projections, Methods Funct. Anal. Topology, 2006, V.12, N 1, 57-73.

[12] Moskaleva Yu.P., On *-representations of the algebra $\mathcal{P}_{4, \text { abo, } \tau}$, Uchenye Zapiski Tavricheskogo Natsional'nogo Universiteta imeni Vernadskogo, Seriya Matem. Mech. Inform. Kibern., 2005, N 1, $27-35$ (in Russian). 